\title{
ETV4 and ETV5 drive synovial sarcoma through cell cycle and DUX4 embryonic pathway control
}

\author{
Joanna DeSalvo, ${ }^{1,2}$ Yuguang Ban, ${ }^{2,3}$ Luyuan Li, ${ }^{1,2}$ Xiaodian Sun, ${ }^{2}$ Zhijie Jiang, ${ }^{4}$ Darcy A. Kerr, ${ }^{5}$ Mahsa Khanlari, ${ }^{5}$ Maria Boulina, ${ }^{6}$ \\ Mario R. Capecchi, ${ }^{7}$ Juha M. Partanen, ${ }^{8}$ Lin Chen, ${ }^{9}$ Tadashi Kondo, ${ }^{10}$ David M. Ornitz, ${ }^{11}$ Jonathan C. Trent, ${ }^{1,2}$ and Josiane E. Eid ${ }^{1,2}$ \\ 'Department of Medicine, Division of Medical Oncology, 25ylvester Comprehensive Cancer Center, and ${ }^{3}$ Department of Public Health Sciences, University of Miami Miller School of Medicine, Miami, Florida, \\ USA. ${ }^{4}$ University of Miami Center for Computational Science, Coral Gables, Florida, USA. ${ }^{5}$ Department of Pathology and ${ }^{6}$ Analytical Imaging Core Facility, Diabetes Research Institute, University of Miami \\ Miller School of Medicine, Miami, Florida, USA. 'Department of Human Genetics, Howard Hughes Medical Institute, University of Utah, Salt Lake City, Utah, USA. ${ }^{8}$ Faculty of Biological and Environmental \\ Sciences, University of Helsinki, Helsinki, Finland. ${ }^{9}$ Center of Bone Metabolism and Repair, Research Institute of Surgery, Daping Hospital, Army Medical University, Chongqing, China. ${ }^{10}$ Division of Rare \\ Cancer Research, National Cancer Center Research Institute, Tokyo, Japan. "Department of Developmental Biology, Washington University School of Medicine, St. Louis, Missouri, USA.
}

\begin{abstract}
Synovial sarcoma is an aggressive malignancy with no effective treatments for patients with metastasis. The synovial sarcoma fusion SS18-SSX, which recruits the SWI/SNF-BAF chromatin remodeling and polycomb repressive complexes, results in epigenetic activation of FGF receptor (FGFR) signaling. In genetic FGFR-knockout models, culture, and xenograft synovial sarcoma models treated with the FGFR inhibitor BGJ398, we show that FGFR1, FGFR2, and FGFR3 were crucial for tumor growth. Transcriptome analyses of BGJ398-treated cells and histological and expression analyses of mouse and human synovial sarcoma tumors revealed prevalent expression of two ETS factors and FGFR targets, ETV4 and ETV5. We further demonstrate that ETV4 and ETV5 acted as drivers of synovial sarcoma growth, most likely through control of the cell cycle. Upon ETV4 and ETV5 knockdown, we observed a striking upregulation of DUX4 and its transcriptional targets that activate the zygotic genome and drive the atrophy program in facioscapulohumeral dystrophy patients. In addition to demonstrating the importance of inhibiting all three FGFRs, the current findings reveal potential nodes of attack for the cancer with the discovery of ETV4 and ETV5 as appropriate biomarkers and molecular targets, and activation of the embryonic DUX4 pathway as a promising approach to block synovial sarcoma tumors.
\end{abstract}

\section{Introduction}

Synovial sarcoma (SS) is a high-grade soft tissue malignancy that disproportionately affects adolescents and young adults. The prognosis of SS patients with advanced disease remains poor, with an approximately $50 \%$ five-year survival rate $(1,2)$. The sarcoma fusions SS18-SSX1 and SS18-SSX2 are distinguished by unique associations with chromatin modifier complexes of opposite functions, SWI/SNF-BAF and polycomb repressive complexes (PRC1 and PRC2). The epigenetic deregulation of gene expression that results from SS18-SSX1/2 interactions is thought to drive SS pathogenesis (3-5). We previously reported on the ability of SS18-SSX2 to reprogram mesenchymal precursor cells and activate a signaling network that controls stem cell self-renewal and differentiation. In this network, we detected the constitutive expression of several fibroblast growth factors (FGFs) and their receptors (FGFRs), indicating the existence of FGF autocrine loops in SS cells $(6,7)$. We further showed that FGFR2, a direct target of SS18-SSX2, promoted growth and expression of neural markers in mesenchymal precursor cells and SS cells (6). Other groups have described an active FGFR pathway that promoted SS cell growth (8).

Conflict of interest: The authors have declared that no conflict of interest exists. Copyright: (5) 2021, American Society for Clinical Investigation.

Submitted: July 1, 2020; Accepted: May 11, 2021; Published: July 1, 2021

Reference information: J Clin Invest. 2021;131(13):e141908.

https://doi.org/10.1172/JCl141908.
FGFR signaling involves a large family of FGF ligands and four FGF receptors (FGFR1-4). It is one of the few pivotal pathways that control embryonic development, from early stages to mesoderm patterning and organogenesis $(9,10)$. It carries out these functions by regulating the proliferation, survival, differentiation, and migration of progenitor cells. FGFR is believed to employ the same developmental functions to promote tumor initiation and progression in cancer (11). Aberrant FGFR signaling has been described in several human malignancies, including sarcomas, and attributed to activating mutations, amplifications, or fusions of FGFR genes. However, several studies have presented deregulated expression of FGFs and FGFRs as a driver of oncogenesis (10-16). Moreover, increased FGFR signaling has been shown to induce resistance to inhibitors of other oncogenic kinases (imatinib), as was demonstrated in KIT-mutated gastrointestinal stromal tumors (17).

The E26 transformation specific (ETS) family of transcription factors includes 28 members that share the ETS domain and bind to the core GGA(A/T) sequence. The PEA3 subset of the ETS family consists of 3 factors, ETV1 (ER81), ETV4 (Pea3), and ETV5 (ERM) (18). These factors play essential roles in embryonic development, including neurogenesis (19), spermatogenesis (20), lung branching (21), and limb bud formation (22). In these events, ETV4 and ETV5 were identified as transcriptional targets of FGFR signaling while simultaneously mediating FGFR target gene expression (9). ETV fusions were discovered in Ewing's sarcoma (EWS-ETV1/4) and prostate cancer (TMPRSS2-ETV1/4/5) translo- 
cations (18). In aggressive undifferentiated round cell sarcomas, ETV4 activation by the CIC-DUX4 (capicua-double homeobox 4) fusion is considered a driver of tumor metastasis (23-27). Studies in several malignancies, such as prostate, breast, lung, liver, and gastric cancers, have implicated ETV4 and ETV5 in their pathogenesis $(18,28,29)$. Moreover, ETV1, ETV4, and ETV5 activation is deemed a marker of poor prognosis $(30,31)$, and a causal factor of resistance to MAPK inhibitors in diverse cancers (32).

DUX4 (double homeobox 4) is an embryonic pioneer transcription factor that drives the activation of the vertebrate zygotic genome (33-35). It is expressed within a narrow window of time, between the 2-cell and 4-cell cleavage stages, after which DUX4 is epigenetically silenced and kept in a tight heterochromatic state in somatic tissues (36). In adults, DUX4 is expressed in testis and the thymus (37). The DUX4 gene is embedded in the $3.3-\mathrm{kb}$ units of the D4Z4 subtelomeric macrosatellite repeats that normally include approximately 11 to 150 such units. Facioscapulohumeral dystrophy (FSHD) is an inherited muscular disorder attributed to misexpression of DUX4. Two epigenetic mechanisms are implicated in DUX4 activation; one is contraction of the D4Z4 repeats to a number 10 or less in FSHD1, and the other is a mutated SMCHD1 (structural maintenance of chromosomes flexible hinge domain-containing protein 1) gene in FSHD2. Both mechanisms lead to chromatin relaxation and derepression of the DUX4 locus $(35,36)$. In FSHD patients, untimely activation of the embryonic program by DUX4 is thought to lead to muscle atrophy and death $(38,39)$. DUX4 was first linked to cancer as part of the transforming CIC-DUX4 sarcoma translocation $(27,40,41)$ and IGH fusions in B cell leukemia (42). A recent study revealed DUX4 as a promoter of immune evasion in several types of cancer (43).

In our quest to clarify the role of FGFR signaling in SS pathogenesis, we uncovered an oncogenic axis that connects the FGFR pathway to the oncogenic ETS factors ETV4 and ETV5, and regulation of the DUX4 embryonic program.

\section{Results}

FGFR1, FGFR2, and FGFR3 are required for SS tumor formation in mice. To elucidate the role of FGFR signaling in SS development, we investigated the effect of FGFR loss on tumor formation in an established SS conditional mouse model, SSM2 Myf5-cre (SM2) (44). In this model, the SS18-SSX2 transgene is expressed in MYF5-positive myoblasts. The SM2 mice were generated as previously described (7). To create triple-mutant SSM2 Myf5-cre Fgfr $(1,2,3)$ knockout mice, homozygous floxed alleles of FGFR1, FGFR2, and FGFR3 $\left(F g f r 1^{f / f l}, F g f r 2^{f / / f l}\right.$, and $\left.F g f r 3^{f / / f l}\right)(45,46)$ were mated with SSM2 and Myf5-cre transgenic mice, and then backcrossed to Fgfr-floxed mice to obtain double mutants SSM2 $\operatorname{Fg} f r(1,2,3)^{f / f l}$ and Myf5-cre $F g f r(1,2,3)^{f l / f l}$. The double mutants were mated to each other and to SSM2 transgenics to produce SSM2 Myf5-cre Fgfr $(1,2,3)^{f t / w t}$ (SMF1,2,3.HET) and SSM2 Myf5-cre $F g f r(1,2,3)^{f l / f l}(\mathrm{SMF} 1,2,3 . \mathrm{HO})$, with 1 or $2 \mathrm{Fgfr}$-floxed alleles, respectively. The breeding scheme is delineated in Supplemental Figure 1 (supplemental material available online with this article; https:// doi.org/10.1172/JCI141908DS1). Generation of all progeny followed Mendelian inheritance and exhibited no sex bias. Importantly, double knockout of each of the three FGFRs did not affect the normal development of MYF5 myoblasts (Supplemental Fig- ure 2). As previously reported, tumors in the SM2 mice arise in the intercostal muscles near the cartilage-bone junction, and they are fully grown by 8 weeks of age (7). Based on these criteria, we analyzed tumor development in the SMF models at 10 weeks of age. In a group of $17 \mathrm{SM} 2$ mice, tumors formed with $100 \%$ penetrance, with 3 tumors or more per mouse (Figure 1A, left graph). We found that deletion of 1 wild-type Fgfr allele decreased tumor incidence to $80 \%$ in 10 SMF1.HET mice, $67 \%$ in 12 SMF2.HET mice, and $83 \%$ in 12 SMF3.HET mice. This was accompanied by a decrease in the average tumor number per mouse. Remarkably, knockout of 2 Fgfr alleles led to substantial attenuation of tumor incidence and number in all 3 groups, as 35\% of 20 SMF1.HO mice, $10 \%$ of 20 SMF2.HO mice, and 35\% of 23 SMF3.HO mice developed visible tumors (Figure 1A). The near-complete inhibition of tumor growth in the SMF2.HO mice implies that FGFR2 signaling is more dominant in the SS model than signaling of the remaining two receptors. However, immunohistochemical (IHC) analysis of 2 tumors isolated from SMF1,2,3.HO mice showed expression of FGFR1 (Figure 1B), FGFR2 (Figure 1C), and FGFR3 (Figure 1D), suggesting leakiness in the $\mathrm{Fg} f$-knockout models. For quantitative analysis of Fgfr expression, we measured Fgfr1,2,3 mRNAs in tumors excised from 2 mice belonging to each group among the SM2, SMF1.HO, SMF2.HET, and SMF3.HO groups. Use of SMF2.HET was due to paucity of SMF2.HO tumor tissues available for RNA extraction. Transcription of the Fgfr genes in the SMF tumor models was compared with their expression in corresponding intercostal tissues taken from 2 control, non-tumor-forming Myf5-cre mice. Quantitative reverse transcription PCR (RT-qPCR) analysis showed that Fgfr1 in the SM2 and SMF1.HO tumors was expressed at levels equivalent to those of control rib tissue, whereas levels of Fgfr2 transcripts were 240- and 220-fold higher in SM2 and SMF2.HET tumors, and those of Fgfr3 were 28- and 44-fold higher in SM2 and SMF3.HO tumors, compared with controls (Figure 1E). The almost equivalent Fgfr expression between the SMF.HO/ HET and SM2 tumors likely reflects leakiness in the Fgfr-knockout models; it also supports the notion that FGFR expression is concomitant with SS tumor development.

In conclusion, results from the genetic Fgfr-knockout models clearly indicate that FGFR1, FGFR2, and FGFR3 are expressed in SS tumors, and they play a crucial role in SS development. They also revealed that complete loss of each FGFR is required for significant inhibition of tumor formation. Interestingly, FGFR2 activation appeared to be exclusive to SS tumors, as we failed to detect measurable Fgfr 2 mRNA in the control rib tissues.

FGFR inhibitor BGJ398 impedes SS tumor cell growth in vitro and in vivo. To verify the clinical significance of tumor suppression in the Fgfr-knockout mice, we asked whether pharmacological inhibition of FGFR signaling in human SS cells would lead to similar effects. For this we chose BGJ398, a selective inhibitor of FGFR1, FGFR2, and FGFR3. BGJ398 was shown to be well tolerated and lacking toxicity in mice and humans (47-49). Two human SS cell lines, SYO-1 and HS-SY-II, which express the SS18-SSX2 and SS18SSX1 fusions, respectively, were treated with incremental doses of BGJ398 (5 to $5000 \mathrm{nM}$ ). The effect of BGJ398 on cell growth was measured after 2 days and 4 days of treatment. In a proliferation assay, the SYO-1 and HS-SY-II cells were highly sensitive to FGFR inhibition, and their growth was significantly decreased 

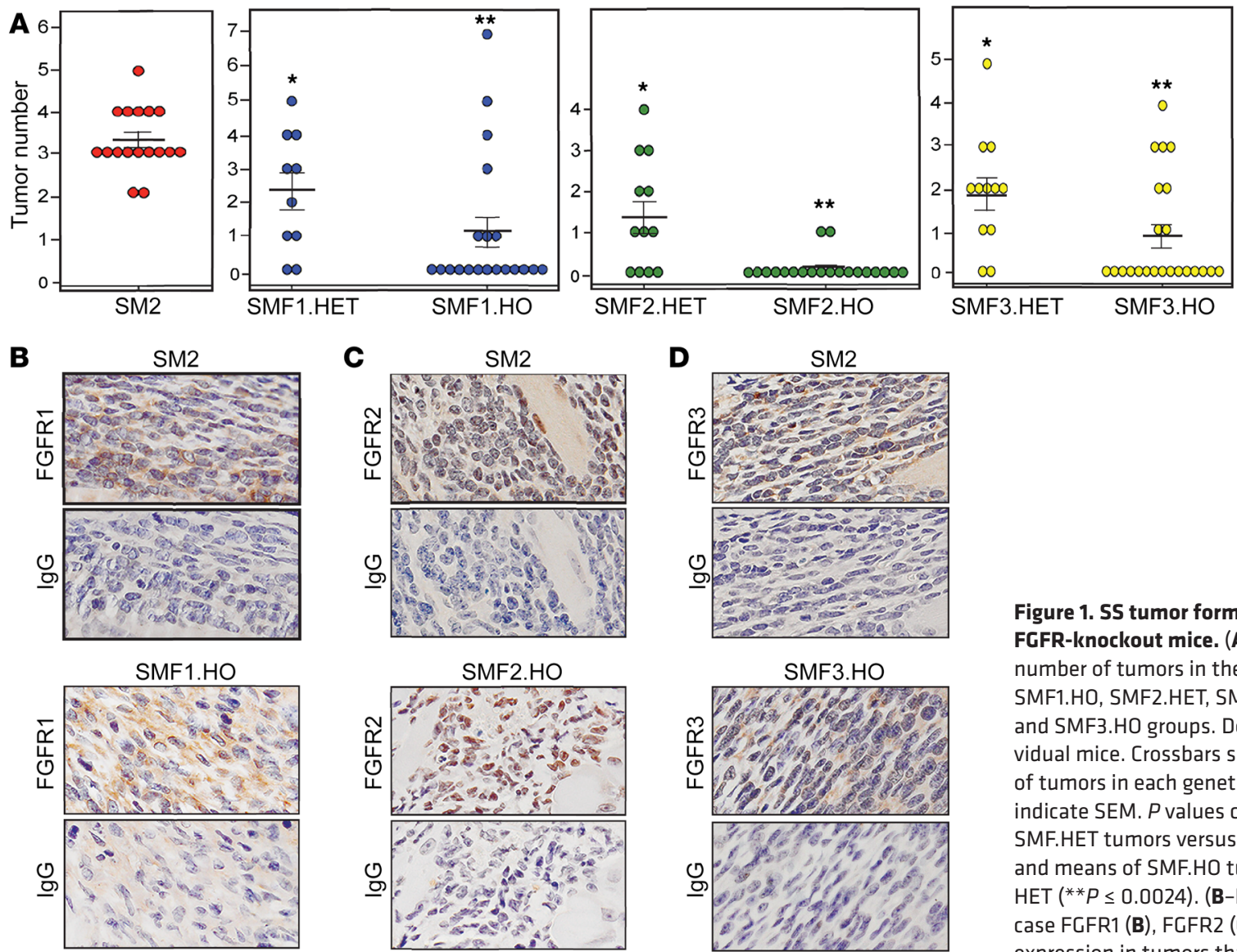

\section{Figure 1. SS tumor formation in}

FGFR-knockout mice. (A) Dot plots show number of tumors in the SM2, SMF1.HET, SMF1.HO, SMF2.HET, SMF2.HO, SMF3.HET, and SMF3.HO groups. Dots represent individual mice. Crossbars show average number of tumors in each genetic model. Error bars indicate SEM. $P$ values compare means of SMF.HET tumors versus SM2 $\left({ }^{*} P \leq 0.00033\right)$ and means of SMF.HO tumors versus SMF. HET ( $\left.{ }^{*} P \leq 0.0024\right)$. (B-D) IHC images showcase FGFR1 (B), FGFR2 (C), and FGFR3 (D) expression in tumors that developed in $\mathrm{SM} 2$, SMF1.HO, SMF2.HO, and SMF3.HO mice. IgC served as background staining. Original magnification, $\times 40$. (E) Box plot shows Fgfr1, $F g f r 2$, and Fgfr3 mRNA levels in the indicated tumor models compared with tissue derived from control mice. Each box represents RNA isolated from 2 mice in each group. The 12 dots in each box indicate individual values normalized against Gapdh and plotted as fold change over control. Data are derived from 2 RT-qPCR experiments performed in 6 replicates. $P$ values compare mean $\mathrm{Fgfr}$ expression in each model against mean expression in control tissues. ${ }^{*} P \leq 0.00014$ $P$ values were calculated using 2-paired $t$ test with $P \leq 0.05$ considered as significant, throughout the paper.

starting with the lowest BGJ398 dose (Figure 2, A and B). To assess the effect of BGJ398 in vivo, SYO-1 cells were subcutaneously implanted in 8 immunodeficient (NU/J) mice. In this xenograft model, SS tumors usually develop with $100 \%$ efficiency. When the SYO-1-derived tumors reached a measurable size, 4 mice were treated with a daily oral dose $(15 \mathrm{mg} / \mathrm{kg})$ of BGJ398, and 4 control mice were treated with vehicle solution, over a 13-day period. Importantly, tumors in all the BGJ398-treated mice failed to grow (Figure 2C). IHC analysis of the BGJ398-treated tumors revealed a 50\% decrease in Ki67 positivity in comparison with the vehicle-treated tumors (Figure 2D). To determine whether FGFR inhibition leads to a similar growth arrest in natural SS tumors, we designated 2 experimental groups, one of 11 and another of 8 tumor-forming SM2 mice, aged 2 months, for similar treatments with BGJ398 (15 mg/kg) and vehicle, respectively. Following the protocol used for the SYO-1 xenografts, we observed that treatment with BGJ398 resulted in a marked decrease in average tumor number (Figure 2E), as well as average tumor load (Figure 2F), per mouse. IHC analysis of 4 tumors from each group showed a $50 \%$ decrease in Ki67-positive cells in the BGJ398-treated SM2 mice compared with vehicle-treated mice (Figure $2 \mathrm{G}$ ). We also observed that the SM2 mice maintained their weight and showed no overt signs of toxicity throughout the treatment period, thus supporting the safety of BGJ398 (Supplemental Figure 3). 
A

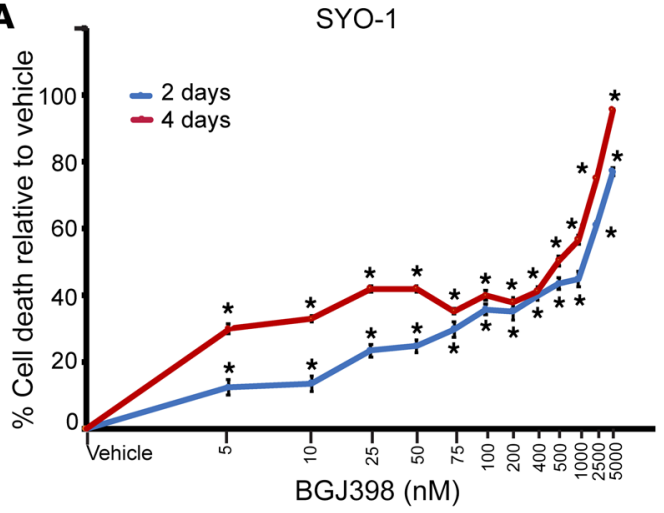

B

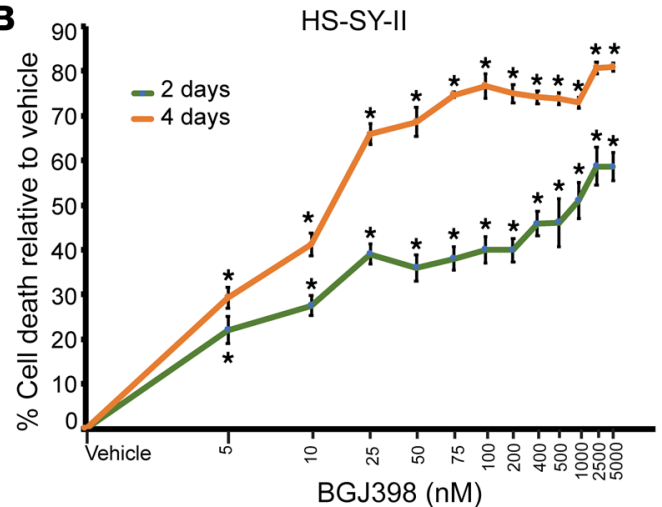

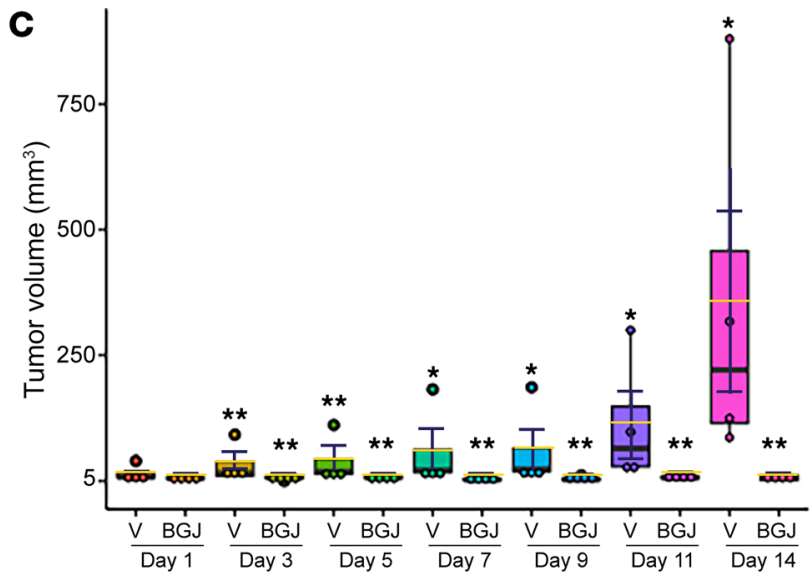

D

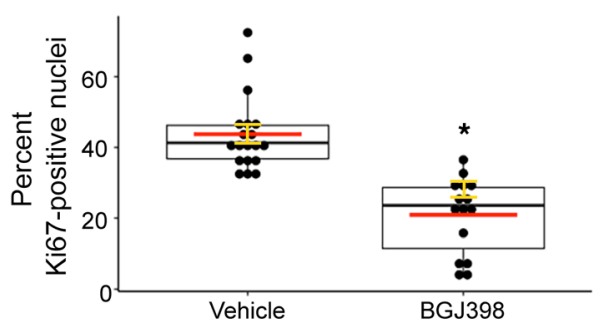

E

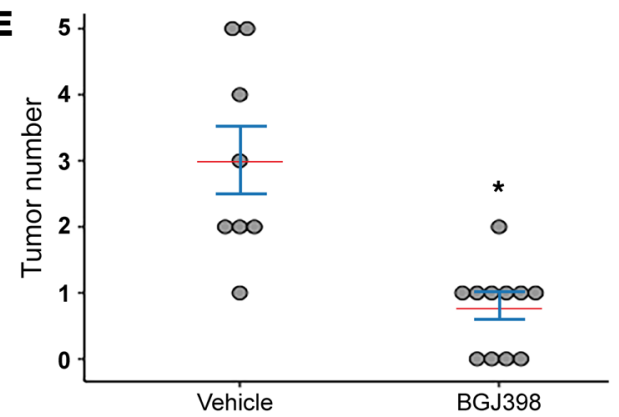

$\mathbf{F}$

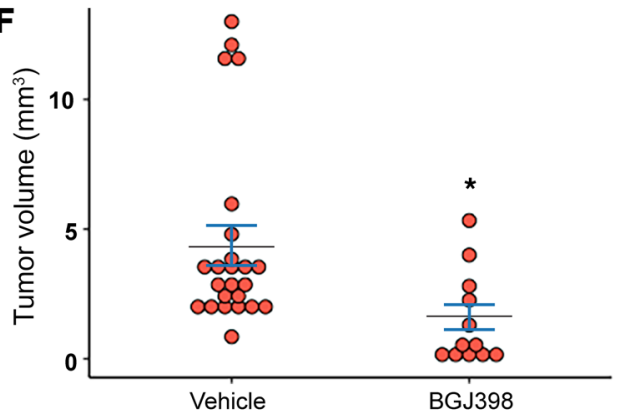

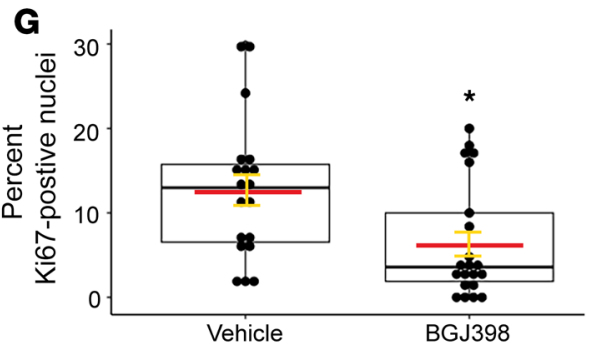

Figure 2. In vitro and in vivo effects of BG)398 in SS cells and tumors. (A and B) Dose-response curves show effects of 2-day and 4-day BG)398 treatments on SYO-1 (A) and HS-SY-II (B) cells relative to vehicle. Each point represents the average of 12 values derived from 2 independent experiments, each conducted in 6 replicates. Error bars indicate SEM. $P$ values $\left({ }^{*} P \leq 0.00079\right.$ in the SYO-1 curves; ${ }^{*} P \leq 0.00191$ in the HS-SY-II curves) compare mean growth of BC)398- and vehicle-treated cells at each point. (C) Box plot shows growth of BC]398-treated and vehicle-treated (V) SYO-1 tumors. Dots represent individual tumors. Crossbars indicate average volumes. Error bars indicate SEM. $P$ values compare mean volumes on days 3-14 after treatment with mean volume on day $1 .{ }^{*} P \leq$ $0.0138 ;{ }^{*} P \geq 0.064$. (D) Box plot shows percentage of Ki67-positive cells in BG|398-treated tumors and vehicle-treated tumors. Overlaid dots represent individual fields counted in 4 tumors from each group; vehicle: 19 fields ( 2925 cells); BGJ398: 15 fields ( 1670 cells). Red crossbars indicate average percentages, and error bars indicate SEM. ${ }^{*} P=0.000194$ was calculated by comparison of mean values of the 2 groups. (E) Dot plot shows tumor number per mouse in vehicleand BC]398-treated SM2 mice. (F) Plot shows individual tumor volumes in vehicle- and BG]398-treated SM2 mice. Crossbars indicate average, and error bars indicate SEM. ${ }^{*} P=0.000937(\mathbf{E})$ and ${ }^{*} P=0.0018$ (F) compare means between the vehicle and BCJ398 groups. (G) Box plot shows percentage of Ki67-positive cells in BGJ398-treated and vehicle-treated SM2 tumors. Dots represent individual fields counted in 4 tumors from each group; vehicle: 19 fields ( 3527 cells); BC]398: 21 fields (3244 cells). Crossbars indicate average percentages, and error bars indicate SEM. ${ }^{*} P=0.00341$ compares means of both groups. 
A

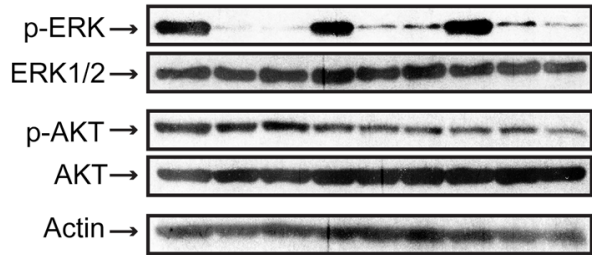

BGJ398 (nM) $\quad \mathrm{V} 50050 \quad \frac{V 50050}{4 \mathrm{~h}} \mathrm{~V} \quad 50050$

D

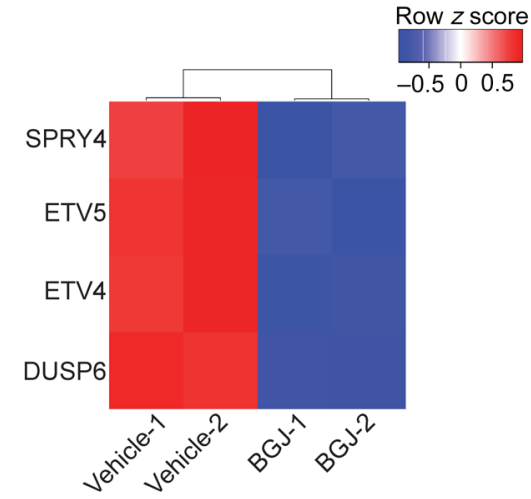

B

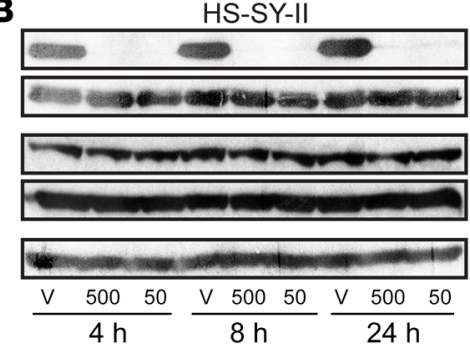

$\mathbf{E}$

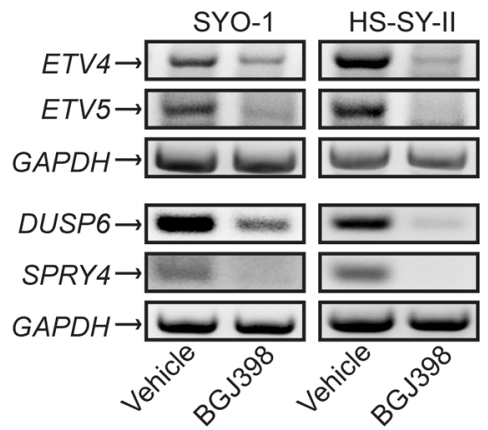

$\mathbf{F}$

G
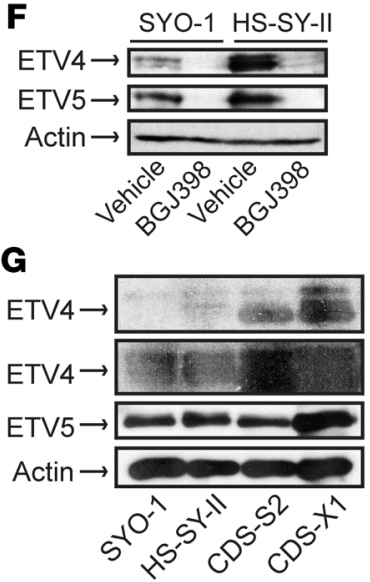

H

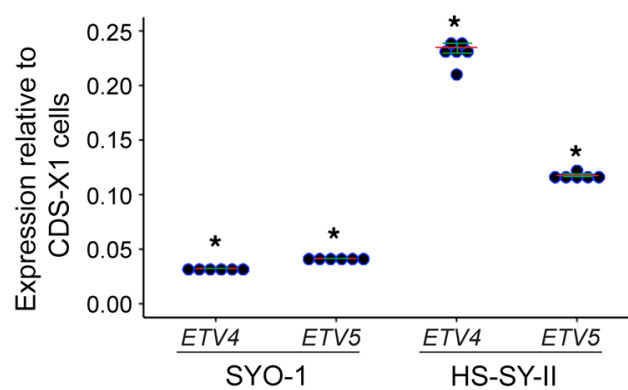

C

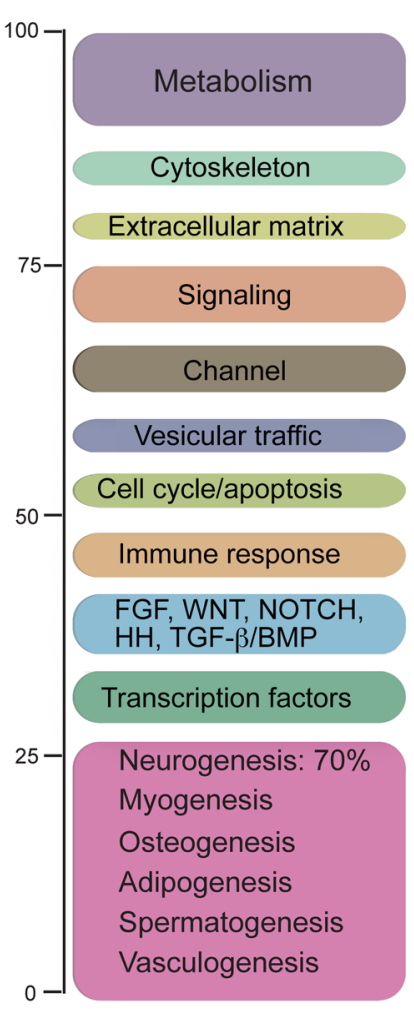

I

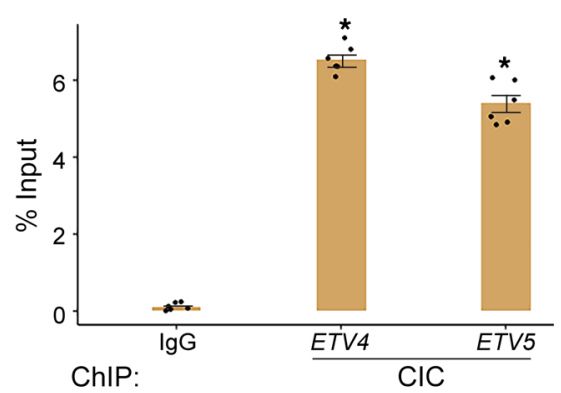

J

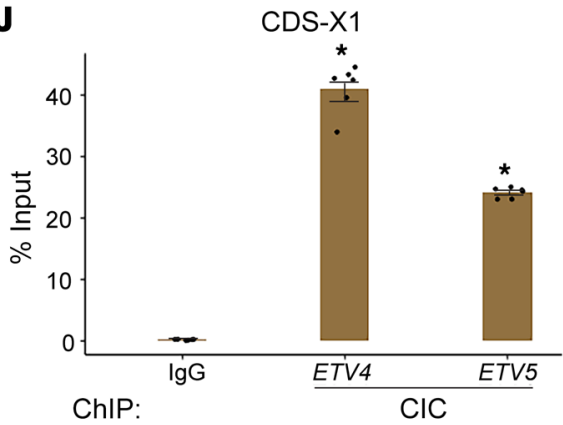

Figure 3. BGJ398 inhibits ETV4 and ETV5 expression in SS cells. (A and B) Immunoblots show the effect of 50 nM and 500 nM BGJ398 on phospho-ERK1/2 and phospho-AKT levels in SYO-1 (A) and HS-SY-II (B) cells at the indicated times. V, vehicle (DMSO). $n=2$. (C) Functional categorization of the SYO-1 transcriptome following a 24-hour BGJ398 treatment. (D) Heatmap illustrates the downregulation of FGFR pathway-related targets by BGJ398 in SYO-1 cells; -1 and -2 represent duplicate samples. (E) RT-PCR of the indicated genes in SYO-1 (left panel) and HS-SY-II (right panel) cells treated with BGJ398 or vehicle. GAPDH served as input control. The PCR primers are described in Methods. (F) Immunoblot shows ETV4 and ETV5 levels in SYO-1 and HS-SY-II cells treated with BGJ398 or vehicle. Actin served as loading control. $n=2$. (C) Immunoblots show ETV4 and ETV5 expression in 50 $\mu$ of SYO-1, HS-SY-II, CDS-S2, and CDS-X1 lysates. Two exposures of the ETV4 immunoblot are included. (H) Dot plot shows ETV4 and ETV5 mRNA levels in SS (SYO-1, HS-SY-II) relative to CDS-X1 cells. Dots represent independent values normalized against GAPDH and plotted as fold change. Data are derived from 2 RT-qPCR experiments performed in triplicate. Crossbars indicate the mean. Error bars indicate SEM. $P$ values $\left({ }^{*} P<0.00001\right)$ compare $\Delta C t$ averages in CDS-X1, SYO-1, and HS-SY-II cells. (I and J) Bar graphs show CIC binding to ETV4 and ETV5 promoters in SYO-1 (I) and CDS-X1 (J) cells. Dots represent independent values from 2 ChIP-qPCR experiments each conducted in triplicate. IgC served as background control. IgG binding and CIC binding were quantified as percentage of input chromatin. Error bars indicate SEM. $P$ values $\left({ }^{*} P \leq 0.0003\right.$ in SYO-1; ${ }^{*} P<0.00001$ in CDS-X1) compare $\Delta$ Ct averages of CIC antibody versus IgG. 
BGJ398 inhibits ERK signaling and ETV4/5 expression in SS cells. Following the growth results, we endeavored to identify the signaling branch of the FGFR pathway regulated by BGJ398 in SS cells. We treated SYO-1 and HS-SY-II cells with $50 \mathrm{nM}$ and $500 \mathrm{nM}$ BGJ398 for 24 hours (before onset of cell death) and harvested the cells at 3 different time points within this period. The two BGJ398 concentrations were chosen based on the dose range that induced 40\%-50\% cell death (Figure 2, A and B). Lysate analysis showed a near-complete depletion of phosphorylated ERK1/2 as early as 4 hours, with either dose of BGJ398, in both SS cell lines (Figure 3, $A$ and $B$ ). The inhibitor failed to alter phospho-AKT levels (Figure 3, A and B), thus indicating that BGJ398 abrogates the MAPK-ERK section of the FGFR pathway without affecting the PI3K pathway. We next sought to analyze the transcription program in SS cells following FGFR inhibition. To this end, total RNA was extracted from SYO-1 cells after a 24-hour treatment with 500 nM BGJ398 and was subjected to RNA-Seq analysis. Five hundred nanomolar was chosen as a safe dose to induce optimal effect on transcription in SS cells within 24 hours. The experiment was performed in duplicate. Transcripts that were significantly regulated $\left(\log _{2}\right.$ fold change $\geq 1.3$ ) in the BGJ398-treated cells were selected and annotated. Functional categorization of the gene targets uncovered mediators of mesenchymal lineage differentiation, with prevalence of neurogenic factors, reflecting the neuronal lineage often described in SS tumors. Furthermore, effectors of developmental pathways, namely FGF, Wnt, NOTCH, HH (hedgehog), and BMP (bone morphogenetic protein), were also represented in the BGJ398-SYO-1 transcriptome (Figure 3C and Supplemental Table 1). However, the most striking finding in the RNA-Seq screen was a marked downregulation of four FGFR signal-related genes: Sprouty homolog 4 (SPRY4), dual-specificity phosphatase 6 (DUSP6), ETV4, and ETV5 (Figure 3D and Supplemental Table 1). SPRY4 and DUSP6 are known negative regulators of FGFR/ MAPK-ERK signaling, and ETV4 and ETV5 belong to the PEA3 subfamily of ETS transcription factors. Decreased transcription of the four genes was validated by RT-PCR in SYO-1 and HS-SYII cells treated with 500 nM BGJ398 (Figure 3E). In addition, BGJ398 treatment led to a distinct attenuation of ETV4 and ETV5 protein expression in both SS cell lines (Figure 3F).

Overexpression of ETV4 and ETV5 is thought to activate the malignant programs in the highly aggressive CIC-DUX4-driven sarcomas (CDS). To begin to understand ETV4/5 regulation in SS cells, we decided to compare expression of the two transcription factors in both sarcoma subtypes. Analysis of lysates with equal protein content derived from SYO-1, HS-SY-II, and two CDS cell lines, CDS-X1 (50) and CDS-S2 (51) showed that ETV4 was present at lower levels in the SS cells while ETV5 concentration was more comparable in the 2 sarcomas (Figure 3G). mRNA measurements in the 4 cell lines showed that ETV4 transcripts in SS cells ranged between $2 \%$ and $23 \%$ of those present in the CDS cells, while SS ETV5 RNAs ranged between $4 \%$ and $12 \%$ of ETV5 transcripts in the CDS cells (Figure $3 \mathrm{H}$ and Supplemental Figure 4A). In normal cells, the tumor suppressor CIC functions as a regulator of ERK signaling and a transcriptional repressor of ETV 4 and ETV5 (52). It controls ETV $4 / 5$ by direct binding to CIC motifs in their promoter regions (53), and when it is mutated in cancer, inactivation of CIC leads to tumor metastasis secondary to ETV4/5 derepression (26,
52). In CDS tumors, fusion of DUX4 C-terminal domain to CIC converts the latter from a repressor to a potent activator of ETV $4 / 5$, thus leading to an aggressive malignancy with poor clinical outcome $(23,27,40,54)$. We immunoprecipitated CIC to compare its expression among the 4 sarcoma lines. The immunoprecipitates revealed significantly lower CIC levels in SS cells compared with CIC-DUX4 in CDS cells (Supplemental Figure 4B). Further analysis showed that ataxin1 (ATXN1) and ataxin1-like (ATXN1L), the two nuclear proteins that normally stabilize the CIC repressor complex, were undetectable in SS cells (Supplemental Figure 4C and data not shown) (52). We next quantified CIC binding to its consensus motifs (53) in ETV4 and ETV5 regulatory regions. We observed that in SYO- 1 cells CIC occupied $6.5 \%$ and $5.4 \%$ of ETV4 and ETV5 chromatin (Figure 3I), and by contrast, CIC-DUX4 was bound to $41 \%$ and $24 \%$ of the respective genes in CDS-X1 cells (Figure $3 \mathrm{~J}$ ). This suggests that, as expected, the majority of active ETV4/5 loci in SS are not bound by CIC, and furthermore, they are derepressed through a mechanism different from CIC-DUX4.

Altogether, our findings have identified ETV4 and ETV5 as active targets downstream of BGJ398 and ERK1/2 in SS cells. Furthermore, ETV4 and ETV5 appear to be activated in SS through a mechanism distinct from their upregulation in CDS cells.

FGFR depletion inhibits SS cell growth in vitro. To examine the effects of individual FGFR inhibition in SS cells, we expressed short hairpin lentiviral vectors targeting FGFR1 (shFGFR1), FGFR2 (shFGFR2), and FGFR3 (shFGFR3) in SYO-1 cells. First, we observed that depletion of one FGFR did not alter the expression of the two remaining receptors (Figure 4, A, C, and E, upper panels). Notably, we found that while ETV4 expression was markedly diminished upon depletion of any one of the three receptors, ETV5 levels were decreased by FGFR2 knockdown alone (Figure $4 \mathrm{C}$, lower panel), and rather enhanced in shFGFR1 cells (Figure 4A, lower panel) and shFGFR3 cells (Figure 4E, lower panel). Our third observation was the extensive cell death caused by the shFGFR vectors: 2 days after infection, $40 \%-60 \%$ of shFGFR1 (Figure 4B), shFGR2 (Figure 4D), and shFGFR3 (Figure 4F) cells were alive compared with cells transduced with control vector (nontarget; TRC2), and 4 days after infection, only $6 \%-17 \%$ in all 3 shFGFR groups survived (Figure 4, B, D, and F).

These findings confirm that signaling from FGFR1, 2, and 3 is required for SS cell growth, and that ETV4 and ETV5 are downstream of the FGFR pathway. The results also suggest that ETV4 and ETV5 are differentially regulated by the individual members of the FGFR family. ETV4 and ETV5 are known targets as well as mediators of FGFR signaling in development $(9,30)$. They also have been linked to several human malignancies. Their dysregulation upon FGFR inhibition in SS cells propelled us to further investigate their role in SS pathogenesis.

ETV4 and ETV5 are activated by SS18-SSX and overexpressed in SS tumors. We and others have reported on autocrine FGF signaling in SS cells. We have also shown that upregulation of various FGF ligands and FGFRs, FGFR2 in particular, is induced by exogenous expression of SS18-SSX in mesenchymal precursor cells (6). To verify whether ETV4/5 expression is concurrent with active FGFR signaling in SS, we began by mining published gene expression arrays of SS18-SSX2-expressing C2C12 myoblasts and human mesenchymal stem cells (7), SS tumors from SSM2 Myf5-cre mice 
A

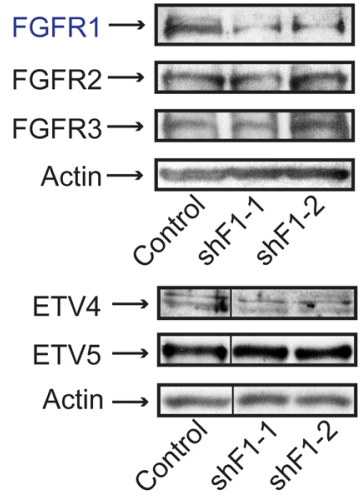

C

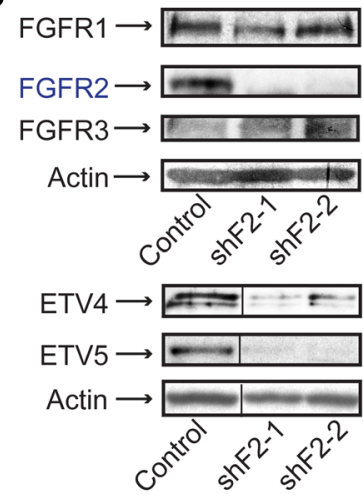

E

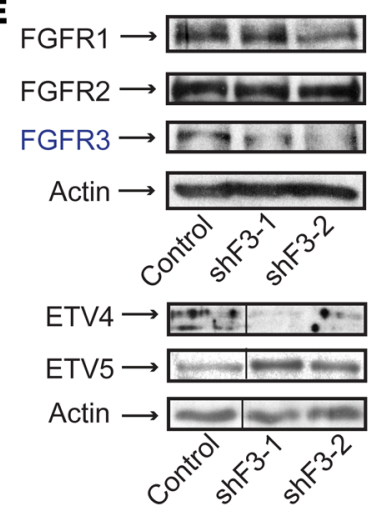

B

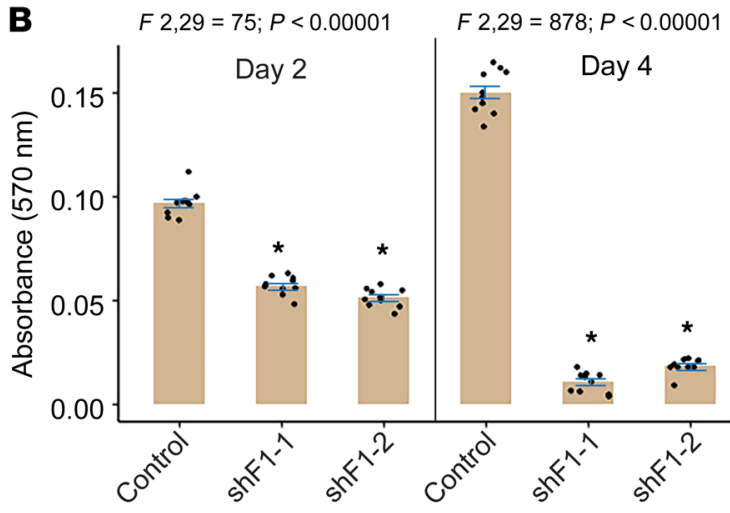

D

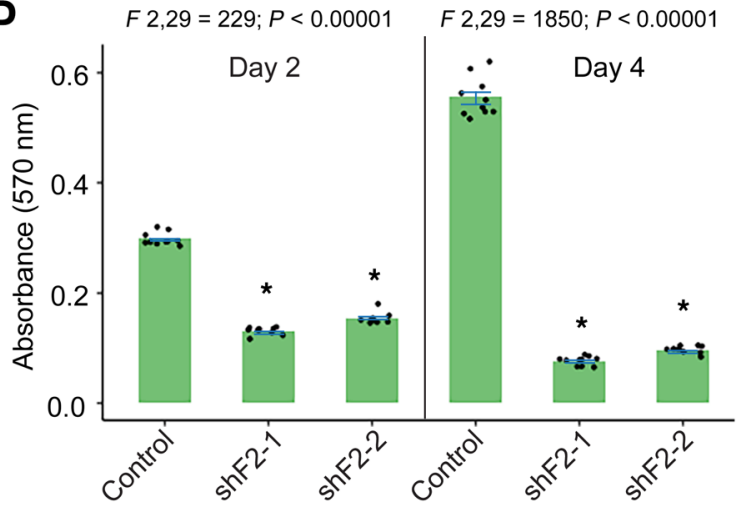

$\mathbf{F}$

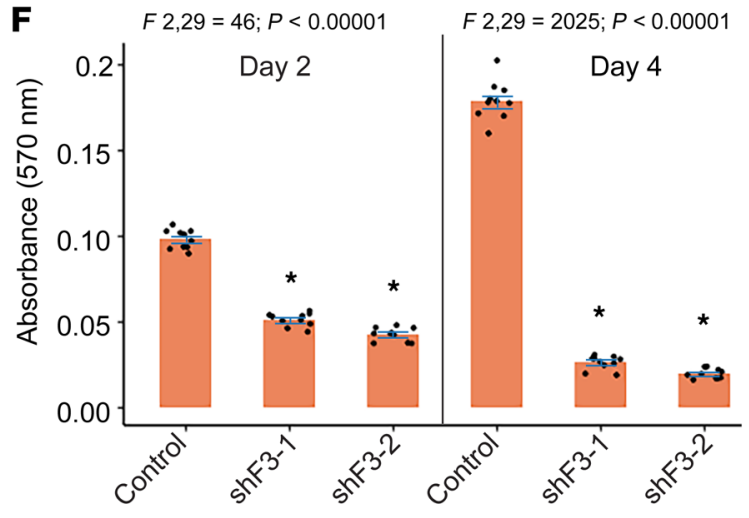

Figure 4. Effect of FGFR depletion on SS cell growth. Immunoblots show FGFR1, FGFR2, FGFR3, ETV4, and ETV5 expression in SYO-1 cells expressing shFGFR1 (A), shFGFR2 (C), and shFGFR3 (E). Control is non-target (TRC2-PLKO.1) vector. shFs represent specific short hairpin FGFR-targeting vectors; -1 and -2 indicate different vectors. Actin served as loading control in all immunoblots. $n=2$. The vertical lines in the ETV4, ETV5, and actin immunoblots designate nonconsecutive lanes of the same SDS-PACE gels. Bar graphs show the effect of FGFR1 (B), FGFR2 (D), and FGFR3 (F) lentiviral depletion on SYO-1 cell growth on day 2 and day 4 after infection. Growth was measured by absorbance at $570 \mathrm{~nm}$. The 10 overlaid dots in each graph represent independent values from 2 sulforhodamine $B$ (SRB) assays conducted in 5 replicates each. Error bars indicate SEM. $P$ values using paired $t$ test compare mean absorbance in each shF group against mean absorbance in the control vector (TRC2) group. ${ }^{*} P<0.00001$ in all graphs. ANOVA $F$ ratios and corresponding $P$ values shown on top of each graph demonstrate significant variation of means among the 3 groups on each day.

SSX2-expressing cells (Figure 5A). To determine the frequency of ETV $4 / 5$ overexpression in human SS, we analyzed tumor tissues from 10 SS patients. IHC revealed robust nuclear ETV4 and ETV5 signals in all 10 tumors (Figure 5, B-D, and Supplemental Figure 5A). Furthermore, the SM2 and SMF1,2,3.HO tumors that displayed detectable levels of FGFR1,2,3 (Figure 1, B-E) were also positive for ETV4 and ETV5 expression (Figure 5, E-G). To gauge ETV4/5 signal intensity against an established positive SS marker, we examined representative human SS, SM2, and SMF tumors for TLE1 expression (57). As expected,

(44), and 2 human SS tissue arrays $(55,56)$. This comprehensive analysis revealed prevalent expression of ETV4 and/or ETV5, several FGFs, and at least one FGFR, in all examined arrays (Supplemental Table 2). Of particular interest, the SSM2 Myf5 tumors showed upregulation of $\mathrm{Fg} f \mathrm{r} 2$ alone, whereas activation of all three FGFRs was detected by IHC and RT-qPCR in our models (Figure $1, \mathrm{~B}-\mathrm{E})$. This discrepancy could be a matter of detection threshold and choice of control tissue, as it corroborates our finding that Fgfr2 expression was highest ( $\geq 220$-fold) among the three FGFRs (Figure 1E) in the SM2 tumors, and given the higher sensitivity of qPCR, we detected in addition Fgfr1 and Fgfr3 mRNAs in the tumors. To test whether ETV4/5 upregulation is dependent on SS18-SSX, we transduced C2C12 myoblasts with a retroviral SS18SSX2 vector and harvested the cells 2 days later. We found that ETV4 and ETV5 levels were significantly increased in the SS18- all tumors tested displayed a strong nuclear TLE1 expression (Supplemental Figure 5B). For better quantitation of ETV4 and ETV5 expression in SS tumors, we measured ETV4/5 mRNA levels in the same tumors (SM2, SMF1.HO, SMF2.HET, and SMF3.HO) and compared them with levels in the $M y f 5$-cre control tissues used in Figure 1E. RT-qPCR analysis showed that ETV4, ETV5, and Tle1 were transcribed at significantly higher levels -130 - to 230 -fold (Figure 5H), 12- to 32-fold (Figure 5I), and 23- to 38-fold (Figure $5 \mathrm{~J}$ ), respectively - in the SMF tumors, compared with their expression in control tissues.

In summary, our findings show that ETV4 and ETV5 are downstream targets of the SS18-SSX oncoprotein and are persistently expressed in SS cells and tumors.

ETV4 and ETV5 are essential for SS cell growth and tumor initiation. Following the discovery of widespread ETV4 and ETV5 
A

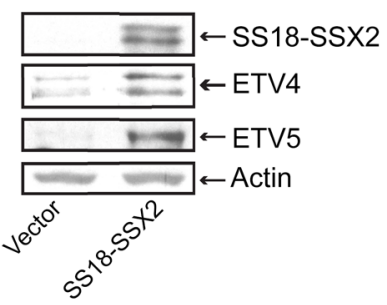

B
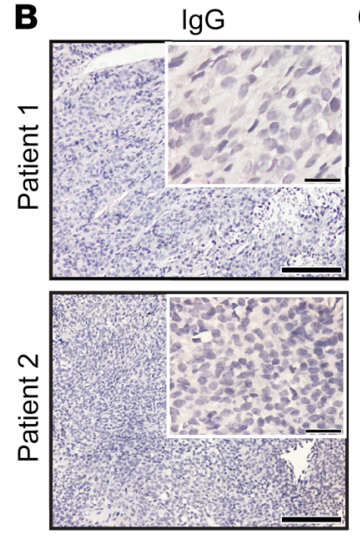

C
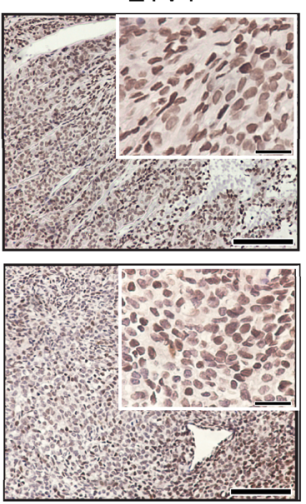

D

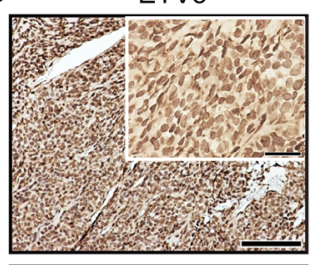

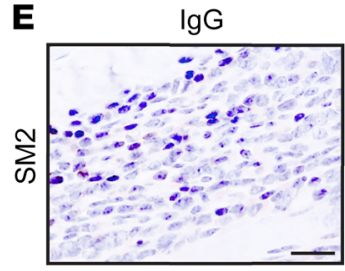
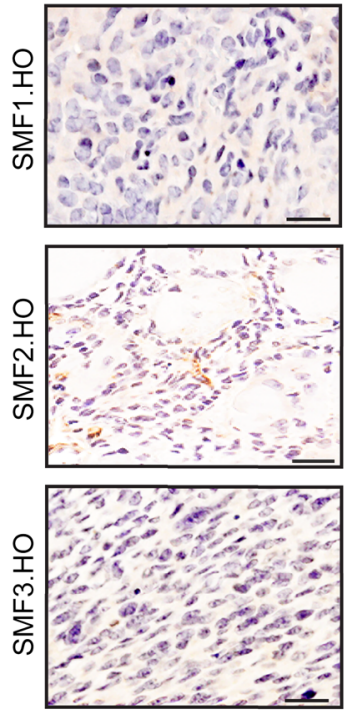
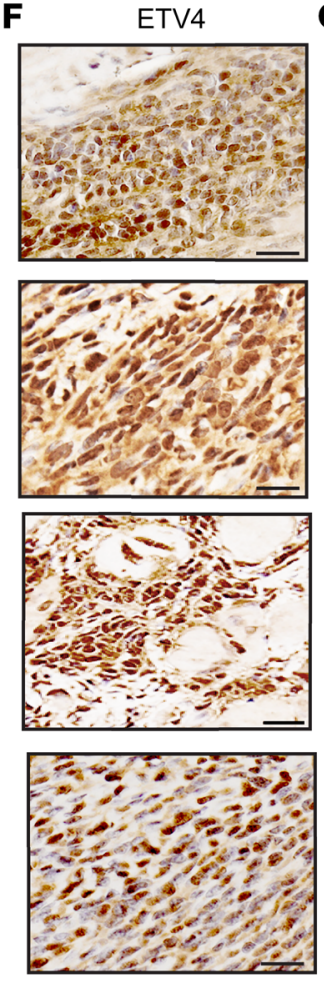

G
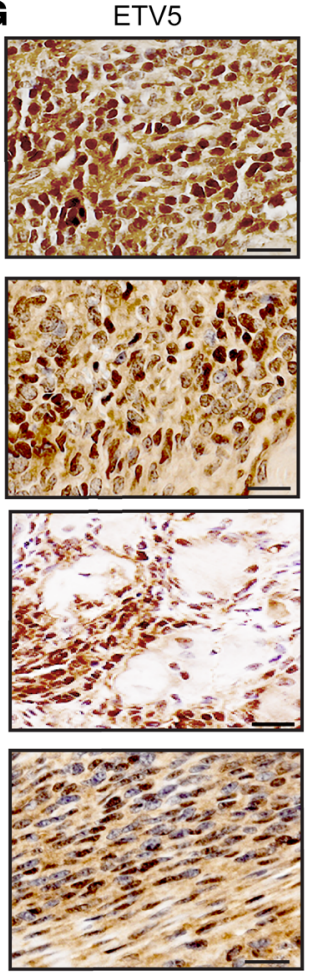

H

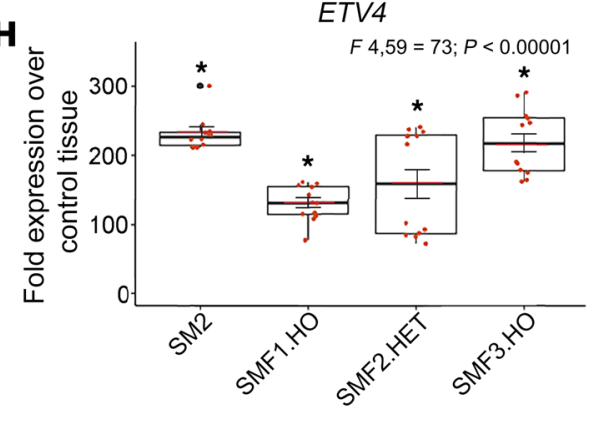

I

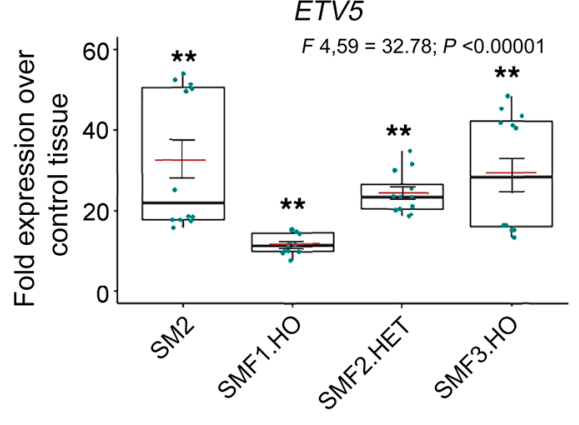

J

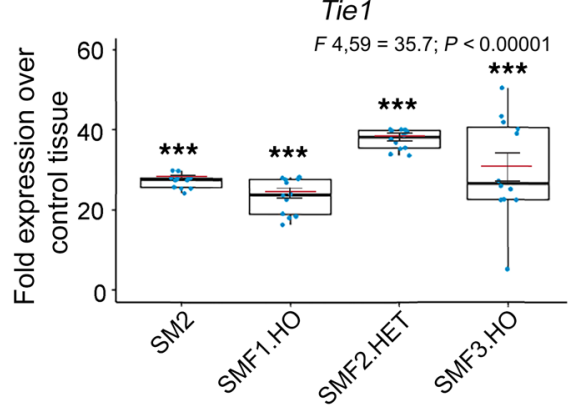

Figure 5. ETV4 and ETV5 expression in SS tumors. (A) Immunoblot shows ETV4 and ETV5 levels in SS18-SSX2-expressing C2C12 myoblasts. Vector is backbone control vector (POZ; see Supplemental Methods). SS18-SSX2 was visualized with anti-FLAG antibody. Actin served as loading control. (B-D) ETV4 and ETV5 IHC staining in tumors resected from two SS patients. Scale bars on the inset images: $50 \mu \mathrm{m}$; all other scale bars: $500 \mu \mathrm{m}$. (E-G) Representative images of ETV4 and ETV5 expression in tumors derived from SM2, SMF1.HO, SMF2.HO, and SMF3.HO mice. Scale bars: $20 \mu \mathrm{m}$. (H-J) Box plots show fold expression of ETV4 (H), ETV5 (I), and Tle1 (J) in the indicated tumor models over tissue derived from control mice. Two mice in each group were used for RNA isolation. The 12 overlaid dots in each box indicate individual values normalized against Gapdh and plotted as fold change over control. Data are derived from 2 RT-qPCR experiments performed in 6 replicates. Red crossbars indicate the mean. Error bars indicate SEM. $P$ values compare average expression in each model against average expression in control tissues. ${ }^{*} P<0.00001 ;{ }^{*} P \leq 0.00065 ;{ }^{* *} P \leq 0.0019$. ANOVA $F$ ratios and corresponding $P$ values shown on top demonstrate significant variation of means among the 4 groups in each plot. 
A

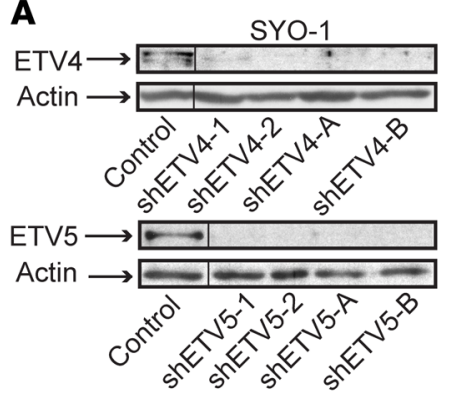

B

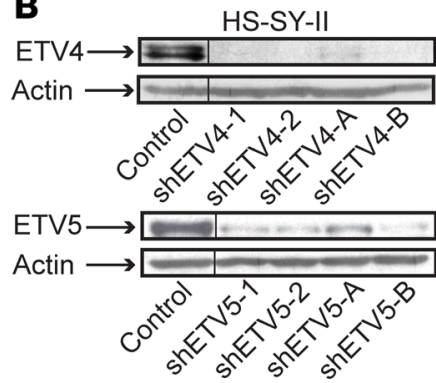

E

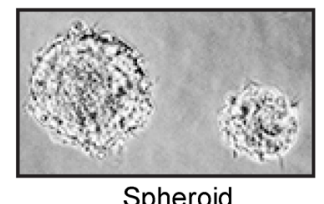

Spheroid

$\mathbf{F}$

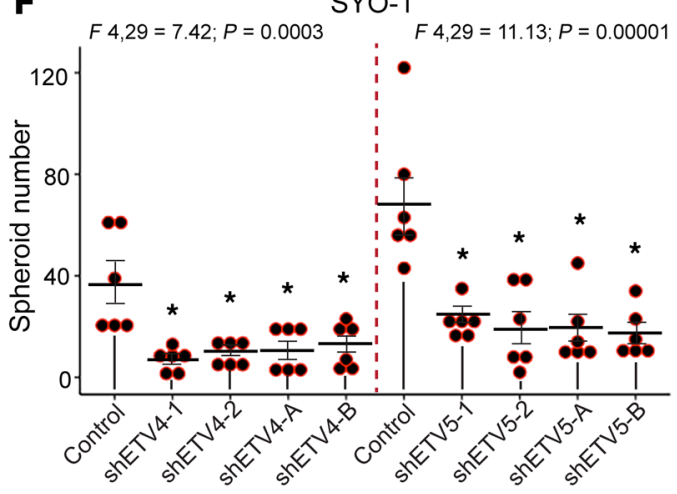

G

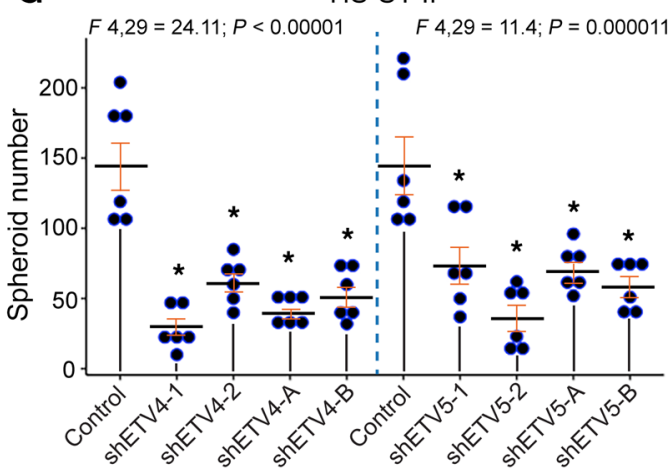

C

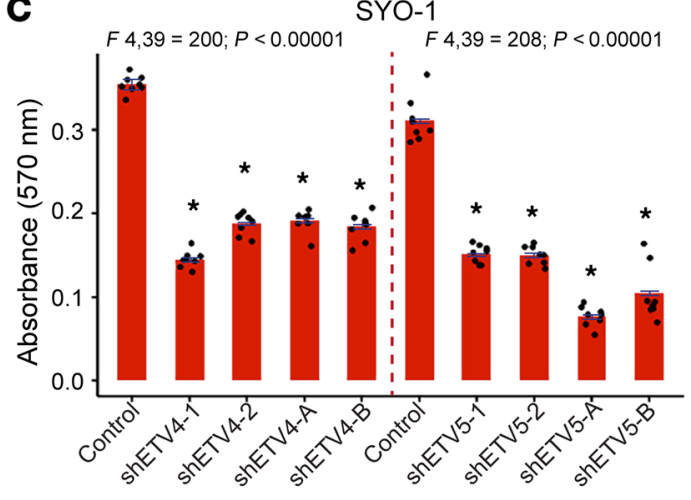

D

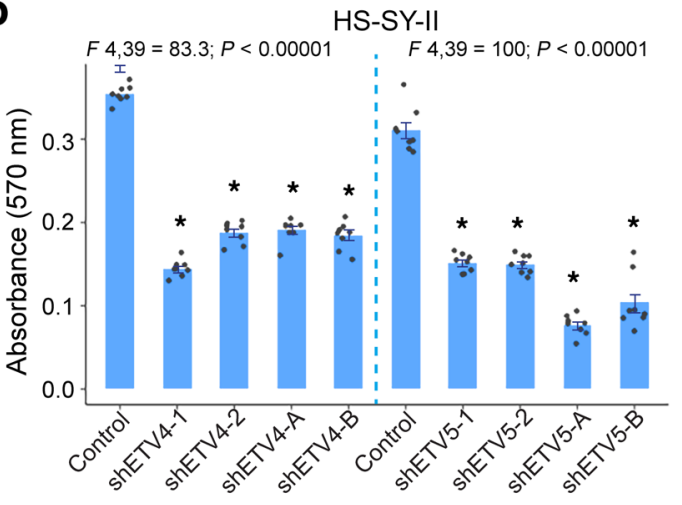

H

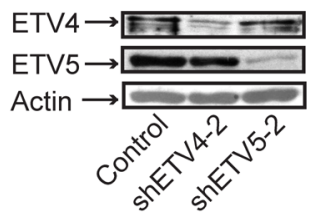

I

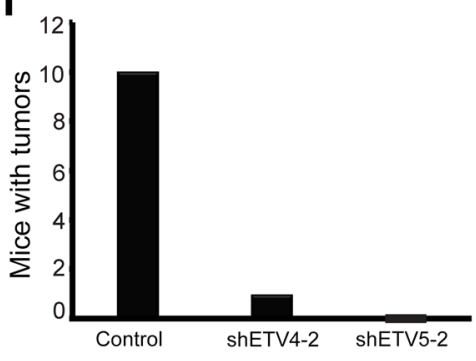

Figure 6. Effect of ETV4 and ETV5 inhibition on SS cell and tumor growth. (A and B) Immunoblots show ETV4 and ETV5 depletion in SYO-1 (A) and HS-SY-II (B) cells. shETV4-1, -2, -A, and $-B$ and shETV5-1, $-2,-A$, and $-B$ designate the lentiviral vectors used for specific knockdown of ETV4 and ETV5, respectively. Control is non-target vector (TRC2-PLKO.1). Dividing lines indicate noncontiguous lanes of the same SDSPAGE gels. (C and $\mathbf{D})$ Bar graphs show the effects of ETV4 and ETV5 lentiviral depletion on SYO-1 (C) and HS-SY-II (D) cell growth. Growth was measured by absorbance at $570 \mathrm{~nm}$. The 8 dots in each graph represent independent values from 2 SRB assays conducted in 4 replicates each. Error bars indicate SEM. $P$ values compare mean absorbance in shETV groups against mean absorbance in the control vector (TRC2) group. ${ }^{*} P<0.00001$ in both graphs. (E) Representative digital image of SS sarcospheres. (F and $\mathbf{G}$ ) Dot plots show numbers of spheroids formed by SYO-1 (F) and HS-SY-II (C) cells expressing shETV4 and shETV5. Dots represent individual values derived from 2 independent assays performed in triplicate. Crossbars indicate the mean, and error bars indicate SEM. $P$ values were measured by comparison of the average of each experimental group with that of the control (TRC2) group. ${ }^{*} P$ range: $0.0318-0.0058$ in SYO- 1 ; ${ }^{*} P$ range: 0.018-0.001 in HS-SY-II. (H) Immunoblot illustrates specific knockdown of ETV4 and ETV5 in SYO-1 cells transduced with shETV4-2 and shETV5-2 and implanted in NU/J mice. (I) Graph shows the total number of mice that developed tumors in each group, 3 months after injection. Actin served as loading control in all immunoblots. ANOVA $F$ ratios and corresponding $P$ values shown on top demonstrate significant variation of means among the 5 groups in each plot. expression in SS, we wished to investigate the extent of their contribution to SS pathogenesis. We addressed this question by using short hairpin lentiviral vectors that specifically target each transcription factor. We found 4 such vectors that resulted in sub- stantial depletion of ETV4 (shETV4-1,2; shETV4-A,B) and ETV5 (shETV5-1,2; shETV5-A,B) in the SYO-1 (Figure 6A) and HS-SY-II (Figure 6B) SS cells. We observed that suppression of either ETV4 or ETV5 led to significant inhibition of SS cell proliferation (Figure 
6, C and D). Furthermore, individual loss of either ETV4 or ETV5 impeded the ability of SYO-1 and HS-SY-II cells to form sarcospheres in serum-free culture media (Figure 6, E-G), implying that both factors are required for the maintenance of SS-initiating cells in vitro. To substantiate this finding in vivo, we subcutaneously implanted SYO-1 cells in immunodeficient mice, 24 hours after their transduction with the non-target (control), shETV4-2, and shETV5-2 vectors. The 24-hour time point was chosen to avoid injection of dying shETV-infected cells. We confirmed by immunoblotting the specific depletion of ETV4 and ETV5 in the injected cells (Figure 6H). This experiment was conducted in 2 parts, with 5 mice per group in each part, hence a total of 10 mice per group. We monitored the mice for tumor growth over a 90-day period. At the end of the 3 months, all 10 control mice and 1 shETV 4 mouse had formed large SS tumors, while none of the shETV5 mice developed a visible mass at the injected site (Figure 6I).

Altogether, the functional results establish ETV4 and ETV5 as drivers of SS growth, and, importantly, both factors seem to be required for this task.

ETV4 and ETV5 control the cell cycle, chromatin structure, and the DUX4 zygotic program in SS cells. To better understand ETV4/5-mediated effects on SS growth, we proceeded to identify the transcriptional programs controlled by ETV4/5 in SS cells. Total RNA was purified from SYO-1 cells, 24 hours after transduction with control, shETV4-2, and shETV5-2 vectors, then was analyzed by RNA-Seq. This experiment was conducted in duplicate. Gene annotation and functional categorization of differentially regulated transcripts (Supplemental Table 3) yielded striking results: a massive downregulation (301 genes) of cell cycle- and DNA repair-related genes in ETV4-depleted (shETV4-2a,2b) and ETV5-depleted (shETV5-2a,2b) cells (Figure 7A and Supplemental Figure 6). The downregulated genes encoded mediators of early and late cell cycle progression, and checkpoint control, including E2F1/2, FOXM1, cyclins, and CDKs (cyclin-dependent kinases). In addition to ETV 4 and ETV5 that were specifically depleted by their respective targeting vectors, we identified a group of 76 genes representing transcription factors that were differentially expressed in the shETV4- and/or shETV5-transduced cells. Worthy of note were the growth and differentiation regulators EGR1 and EGR2 (early growth response 1 and 2) and several members of the AP1 family, such as JUN, FOS, FOSB, FOSL1, and FOSL2, all activated upon ETV4 and ETV5 loss (Figure 7B and Supplemental Figure 7). A third category of interest included 135 genes of chromatin structure modulators (Figure 7C and Supplemental Figure 7). Functional classification of this group revealed a noticeable pattern of chromatin opening illustrated by downregulation of heterochromatin-promoting chaperones and DNA and histone methyltransferases (DNMT1/3B, UHRF1, CBX1/5, SUV39H1/2, EZH2, CHAF1A/B) and a contrasting upregulation of histone demethylases (KDM4B/ $E / F)$. An unexpected finding that attracted our attention was the evident activation of the DUX4-driven zygotic program in the shETV4 as well as the shETV5 cells, the same program shown to be induced by DUX4 in the FSHD atrophic muscles $(34,35)$. We identified 33 upregulated members of the DUX4 pathway, including DUX4, ZSCAN4, LEUTX, CCNA1, and the PRAME, TRIM, and $M B D 3 L$ families (Figure 7D). We validated by RT-PCR a representative selection of genes from each of the 4 pathways (Figure 8).
Together, these data implicate ETV4 and ETV5 as pervasive promoters of cell cycle progression in SS cells. Moreover, it appears that the two factors impose a state of repressed chromatin in SS cells, including silencing of the zygotic program controlled by DUX4.

Convergence of ETV4/5 and DUX 4 in SS cells. To further examine the role of ETV4/5 in cell cycle progression, we expressed shETV4-2 and shETV5-2 vectors in SYO-1 and HS-SY-II cells. Lysate analysis showed a significant attenuation in the levels of the cell cycle master controller E2F1 in both cell lines. Interestingly, while E2F1 was decreased upon ETV4 and ETV5 depletion in HS-SY-II cells, we repeatedly observed that in SYO-1 cells, E2F1 was affected by shETV5 alone (Figure 9A). Notably, levels of the cyclin-dependent kinase inhibitor CDKN1A (p21), the growth suppressor EGR1, and FOS polypeptides (detected by an antibody that recognizes c-FOS, FOSB, FOSL1, and FOSL2) were increased after ETV4 and/or ETV5 loss in both cell lines (Figure 9A). The next question we addressed was whether DUX4 upregulation contributed to the cell cycle arrest that resulted from ETV4/5 knockdown. To this end we performed combined infections whereby we transduced SYO-1 cells with equal titers of shETV5-2 and 1 of 2 shDUX4 lentiviral vectors (shDUX4-1, shDUX4-2). In this experiment we observed that infection with full shETV5-2 titer, or a mixture of half shETV5 titer and half control titer, led to increased DUX4 protein levels and severe growth arrest, in comparison with SYO-1 cells that were infected with a full titer of control vector. The latter group were almost devoid of DUX4 (lanes 1-3 in Figure 9, B and C). When shETV5 and shDUX4 were coexpressed at half titer each, we observed a significant rescue of the growth arrest phenotype. This was accompanied by a measurable depletion of DUX4 (lanes 4 and 5 in Figure 9, B and C). Importantly, levels of ETV5 depletion in all 4 shETV5 groups included in this experiment were unchanged (Figure 9B). These data support the notion that activated DUX4 contributes to the proliferation arrest induced by shETV4/5. To analyze the direct effects of DUX4 activation in SS cells, we transduced SYO-1 and HS-SY-II cells with a doxycycline-inducible DUX4 expression vector (58). DUX4 induction led to extensive cell death in both lines (Figure 9, D and E). RT-PCR analysis of RNA purified from DUX4-expressing SYO1 cells 16-24 hours after induction (harvested before extensive cell death) confirmed the upregulation of known DUX4 targets, including ZSCAN4, MBD3L3, KDM4E, and CCNA1. In addition, transcripts of CDKN1A, EGR1, and FOS family members were upregulated in the DUX4-expressing cells compared with uninduced cells (Figure 9F). We found that the transcriptional activation of the four genes was accompanied by significant elevation in the levels of their encoded proteins in DUX4-expressing SYO-1 and HS-SY-II cells (Figure 9G). By contrast, DUX4 expression failed to alter E2F1 and SS18-SSX levels (Supplemental Figure 8), suggesting that DUX4 acts downstream of both proteins in SS cells. With these results we conclude that upregulation of the growth controllers (CDKN1A, EGR1, FOS) seen in the shETV4/5 cells is most likely driven by active DUX4. CDKN1A and EGR1 activation by DUX4 was previously reported $(59,60)$, and both growth suppressors have been implicated in mediating the DUX4-dependent atrophy in FSHD muscles.

Genomic ETV4/5 interactions in SS cells. The majority of 301 cell cycle- and DNA repair-related genes identified in the shETV4/5 
A

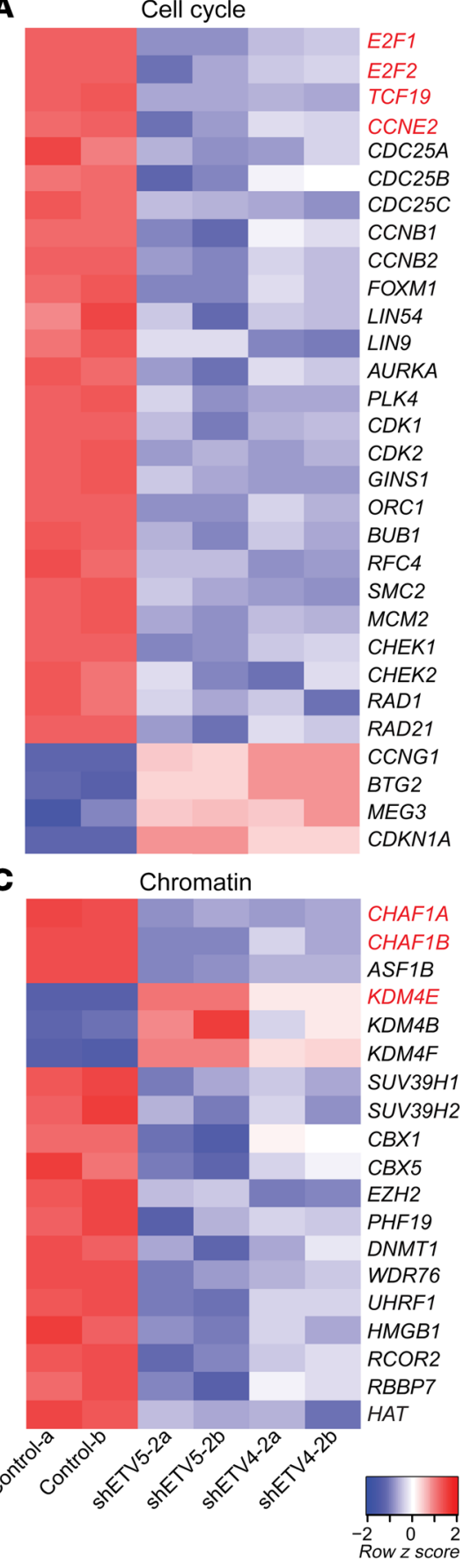

B

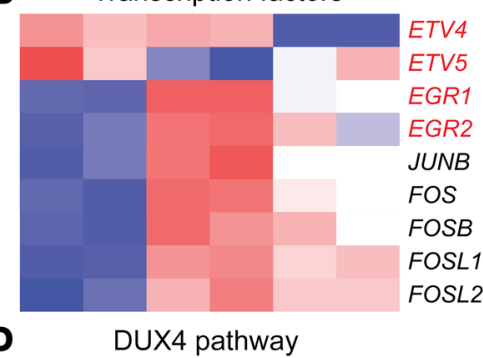

D

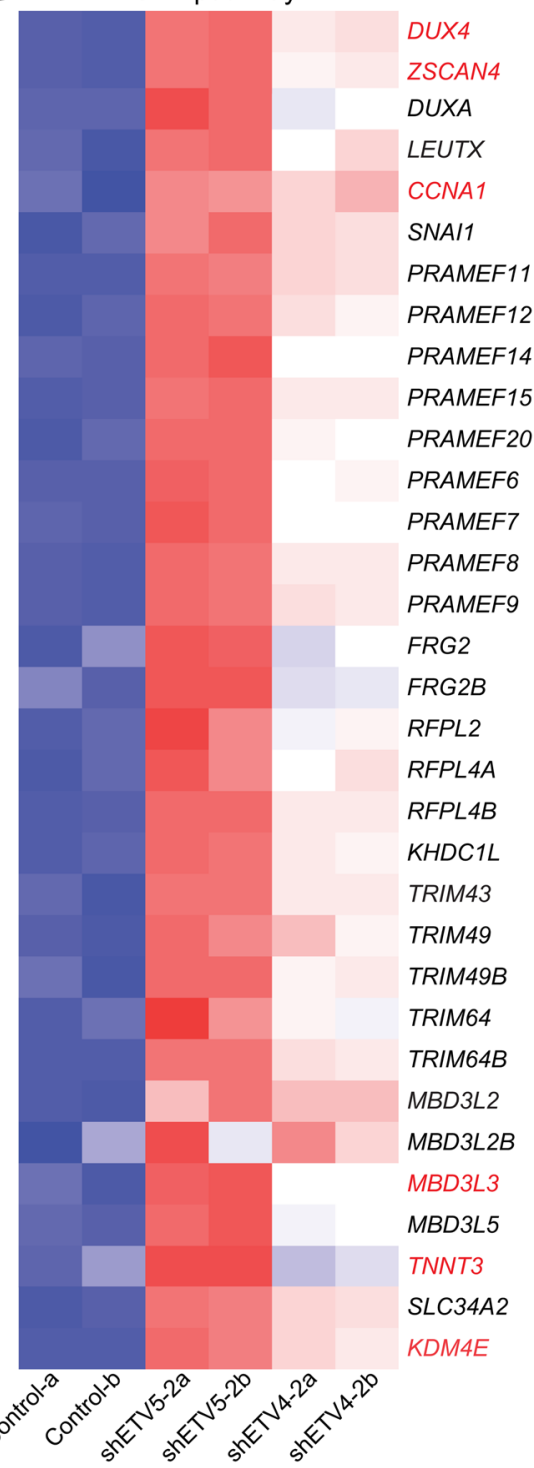

Figure 7. Analysis of the SS cell transcriptome upon ETV4 and ETV5 knockdown. Heatmaps of differentially expressed genes in SYO-1 cells expressing control, shETV4-2, and shETV5-2 vectors. Lanes $a$ and $b$ represent duplicate RNA samples. A selection of genes belonging to the cell cycle (A), transcription factors (B), chromatin (C), and DUX4 pathways (D) is shown.

RNA-Seq screen are recognized as transcriptional targets of the (retinoblastoma) Rb-E2F complex $(61,62)$. To clarify the mechanism by which ETV4/5 knockdown resulted in the downregulation of these genes, we inquired whether the ETV factors partnered with the E2F factors to promote their transcription. Since ETV4 and ETV5 appeared to function similarly in SS cells, we chose to

conduct the following experiments with ETV5, as it appears to be more abundant than ETV4 in SYO-1 cells, and also for the availability of a specific antibody that efficiently pulls down ETV5. To explore ETV5E2F1 interactions, we performed ETV5 and E2F1 pull-downs in SYO-1 cells and re-immunoprecipitated the former with a specific E2F1 antibody (Supplemental Figure 9A) and the latter with anti-ETV5 antibody (Supplemental Figure 9B). In these assays, we failed to detect a direct association between ETV5 and E2F1. We then asked whether ETV5 directly regulated E2F1 and searched for potential ETV5 binding sites on the $E 2 F 1$ locus. We looked for motifs that fit within the $(\mathrm{A} / \mathrm{C}) \mathrm{GGA}(\mathrm{A} / \mathrm{T})$ GT module $(63,64)$. Sequence analysis of the $E 2 F 1$ gene (NCBI Ensembl; http:// useast.ensembl.org/Homo_sapiens/Gene/ Summary) extending $10 \mathrm{~kb}$ upstream and downstream of the transcriptional start site (TSS) identified a candidate ETV5 binding motif, AGGAAGT, situated approximately $1 \mathrm{~kb}$ upstream of the TSS (Figure 10A).

DUX4 is expressed between the 2-cell and the 4-cell embryonic stage, after which it is tightly silenced throughout development. Elegant studies have shown that the chromatin assembly factor $1 \mathrm{~A} / \mathrm{B}(\mathrm{CHAF1A} / \mathrm{B})$ complex is a major chromatin repressive complex that keeps the DUX4 gene in a silenced state (65). In another study, CHAF1A/B block was described as essential to DUX4 activation of the zygotic genome, and knockdown of either CHAF1A or CHAF1B abrogated DUX4 function (34). CHAF1A and CHAF1B play a crucial role in chromatin assembly during DNA replication and DNA repair, and they are regulated by Rb-E2F (61, 62). Moreover, transcription of CHAF1A and CHAF1B was downregulated in the shETV4/5 SS cells (Figure 7C and Figure 8). Coimmunoprecipitation experiments in SYO-1 cells showed that CHAF1A exists in a complex with CHAF1B but does not interact with either ETV5 or E2F1 (Supplemental Figure 9, C and D). To examine E2F1 behavior in SYO-1 cells, we chose two of its established target genes, CCNE2 and CHAF1B. Our search for E2F1 binding motifs that fit the $\operatorname{TTT}(\mathrm{C} / \mathrm{G})(\mathrm{C} / \mathrm{G}) \mathrm{CG}(\mathrm{C} / \mathrm{G})$ sequence (66) identified 1 potential site in the proximity of the CCNE2 TSS (Figure 10D), and 1 site located approximately $2.9 \mathrm{~kb}$ downstream of the CHAF1B TSS (Figure 10G). We performed chromatin immunoprecipitation (ChIP) in naive SYO-1 cells to measure ETV5 and E2F1 binding 

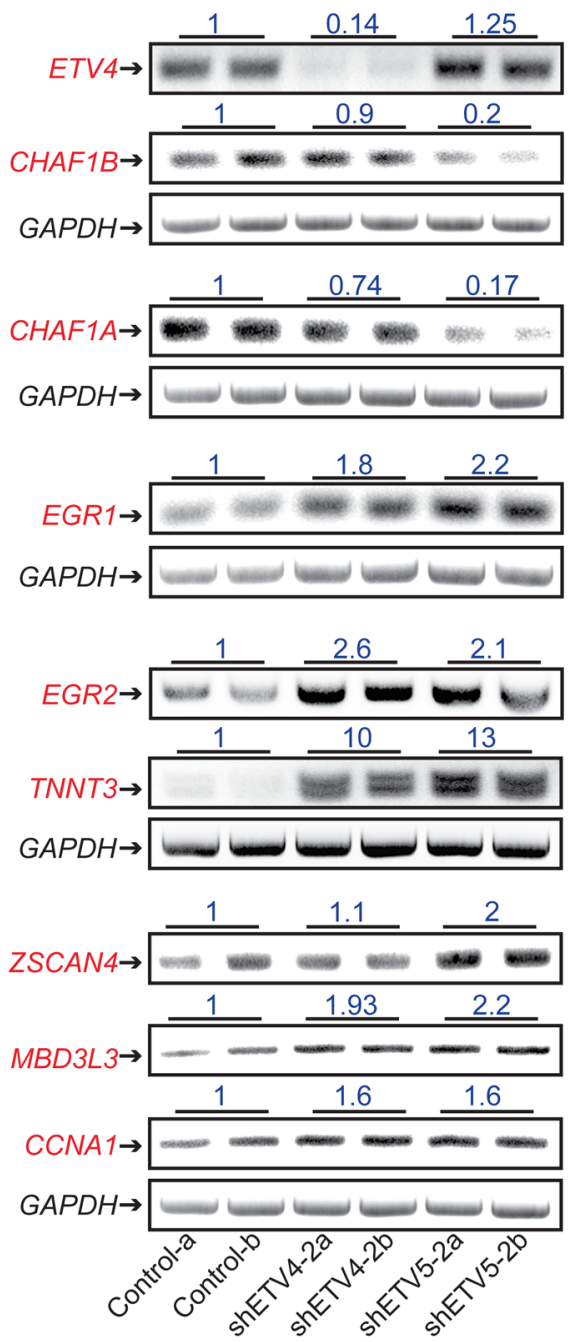
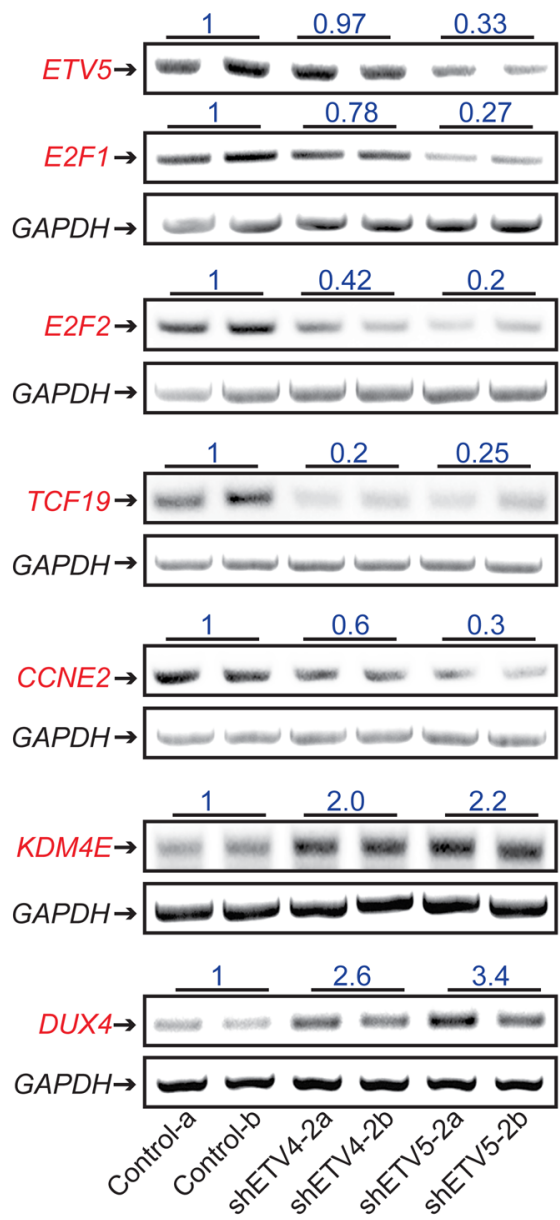

Figure 8. Validation of the SS cell transcriptome upon ETV4 and ETV5 knockdown. RT-PCR of the indicated targets in SYO-1 cells. GAPDH served as input control. RT-PCR was performed on 2 independent RNA samples ( $a$ and $b$ ) of control, shETV4-2-expressing, and shETV5-2expressing cells. Numbers represent band intensities relative to control. Band intensities were calibrated with $G A P D H$; their measurement and the PCR primers used are described in Methods (Statistics) and Supplemental Methods. to the 3 genomic sites (Figure 10, A, D, and G). qPCR analysis showed significant occupancy of the specified regions in comparison with negative IgG controls (Figure 10B and lanes 1 and 2 in Figure 10, E and H). Importantly, E2F1-ChIP conducted in shETV5-2-transduced SYO-1 cells showed a significant decrease in E2F1 binding to CCNE2 and CHAF1B relative to cells transduced with control (non-target) vector (lanes 3 and 4 in Figure 10, E and $\mathrm{H}$ ). To verify whether the 3 binding regions drive endogenous transcription in SS cells, we subcloned the 3 genomic sequences shown in the diagrams in Figure 10, A, D, and G, in the PGL3 luciferase reporter vector (a gift from Agata Levay, University of Miami). The generated vectors were PG-E2F1, PG-CCNE2, and PG-CHAF1B, respectively. When tested in a luciferase reporter assay, all 3 regions appeared to drive endogenous transcription (lane 1 in Figure 10, C, F, and I). To ascertain whether the putative ETV5 and E2F1 binding motifs described above are required for transcription, we deleted the 3 motifs (underlined sequences in Figure 10, A, D, and G) from the cloned regions, and the deletion mutants thus created (PG-dlE2F1, PG-dlCCNE2, PG-dlCHAF1B) were tested for driving transcription. Results from reporter assays showed that removing any 1 of the 3 motifs led to significant attenuation of the luciferase activity driven by their respective unmutated regions (lane 2 in Figure 10, C, F, and I).
These findings strongly suggest that ETV4 and ETV5 are active modulators of the E2F1 pathway in SS cells, and they fit a functional model (Figure 10J) where autocrine FGF/FGFR signaling initiated by SS18-SSX activates ETV4 and ETV5. The two factors trigger E2F and promote cell cycle progression and CHAF1A/B expression, leading to suppression of the DUX4 program. ETV4 or ETV5 depletion results in reversal of this oncogenic axis, DUX4 activation, and cell death.

\section{Discussion}

In the present report we reveal the prevalent expression of the oncogenic ETS factors ETV4 and ETV5 in synovial sarcoma tumors, and their key role as drivers of the cancer. In addition, we have uncovered a regulation of the DUX4 pathway by ETV4/5. Importantly, DUX4 activation led to cell death, thus opening a potential therapeutic path for SS.

Synovial sarcoma, like other mesenchymal cancers, diverts normal developmental pathways to promote its own initiation. FGFR signaling is such a pathway. Interestingly, in the transgenic SS models, FGFR1, FGFR2, and FGFR3 each appear to contribute to tumor formation. This finding was not surprising since SS arises in mesenchymal stem cells (MSCs) (67) that are capable of differentiating into multiple lineages, including bone, 
A
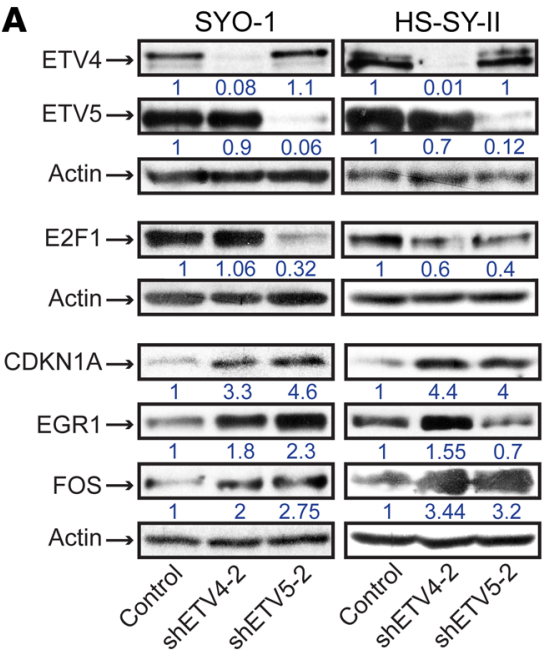

D

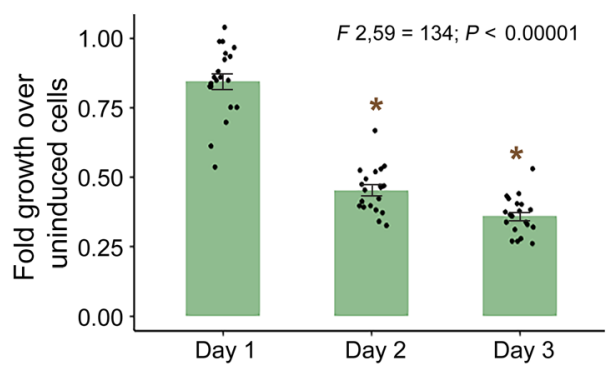

E

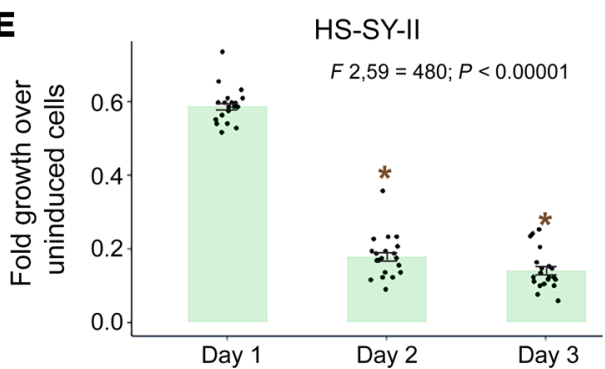

G

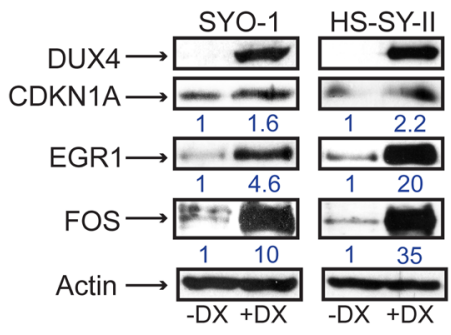

B
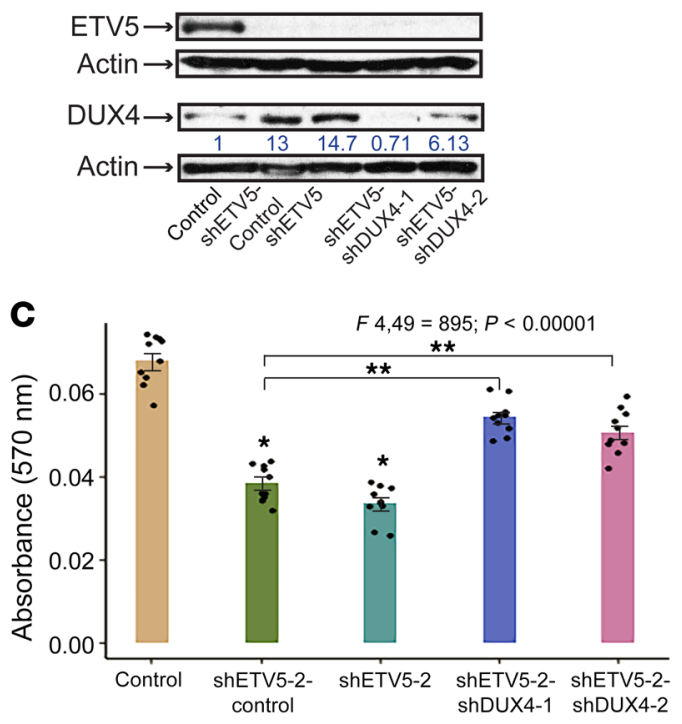

$\mathbf{F}$
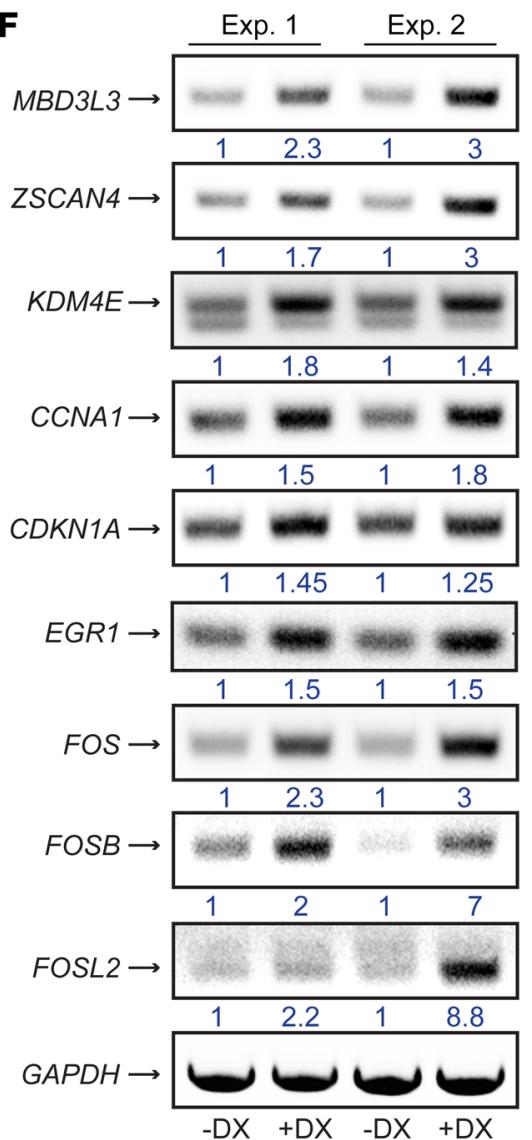

Figure 9. Intersection of ETV4/5 and the DUX4 pathway in SS cells.

(A) Immunoblots show expression of the designated proteins in SYO-1 and HS-SY-II cells expressing control, shETV4-2, and shETV5-2. Numbers represent relative protein signals. Protein intensities were adjusted to actin as described in Statistics. (B) Immunoblots show ETV5 and DUX4 levels in SYO-1 cells expressing shETV5 and shDUX4 vectors. Numbers display relative band intensities compared with actin. (C) Bar graph compares effects of cotransduced shETV 5 and shDUX4 (shETV5-2-shDUX4-1; shETV5-2shDUX4-2), shETV5-2, and control vectors on SYO-1 cell growth. Growth was measured by absorbance at $570 \mathrm{~nm}$. The 10 overlaid dots in each graph represent independent values from 2 SRB assays performed in 5 replicates. Error bars indicate SEM. $P$ values compare mean absorbance between the shETV 5 groups and the control group $\left({ }^{*} P<0.0003\right)$, and between the shETV5-shDUX4 groups and the shETV5-control group $\left({ }^{*} P<\right.$ 0.00001). ( $\mathbf{D}$ and $\mathbf{E}$ ) Fold growth of SYO-1 and HS-SY-II cells expressing lentiviral DUX4 over uninduced cells. Time points indicate days after DUX4 induction with 1 $\mu \mathrm{g} / \mathrm{mL}$ doxycycline. The 20 overlaid dots in every graph represent values from 2 SRB assays performed in 10 replicates each. Error bars indicate SEM. $P$ values compare mean growth on day 2 and day 3 against average growth on day $1 .{ }^{*} P<$ 0.00001 . (F) RT-PCR of gene targets in SYO-1 cells expressing inducible DUX4. Results are shown from 2 independent experiments (Exp. 1 and 2), comparing induced with uninduced cells. DX, doxycycline. Numbers represent ratios of band intensity in induced over uninduced cells. Measurements were adjusted using GAPDH signal as described in Statistics. PCR primers are described in Supplemental Methods. (C) Immunoblot shows proteins activated upon DUX4 induction (+DX) in SYO-1 and HS-SY-II cells. Actin is loading control. ANOVA $F$ ratios and corresponding $P$ values shown on top of the graphs in C-E demonstrate significant variation of means among the 5 and 3 groups, respectively. fat, muscle, and neurons. Thus, the developmental pathways that specify such lineages, and of which FGFR1-FGFR3 are key components, are epigenetically activated in MSCs and ready to signal when required (68). One of the important functions of the SS18-SSX fusion is its ability to reprogram MSCs and activate the neurogenic and osteogenic lineages (6). SS18-SSX fulfills these tasks through recruitment to polycomb-silenced chromatin and disruption of polycomb complexes $(3,4,69)$. Accordingly, the three tightly regulated FGFR receptors that are master controllers of bone and nervous system development $(9,10,70)$ are likely epigenetic targets of SS18-SSX (6). Since the human SS tumors express at least two of the three receptors, a selective inhibitor that blocks FGFR1-3 would in principle have an optimal effect in SS patients. Although in specific developmental settings such as lung branching, FGFR class dictates mediation of proliferation versus differentiation (71), the diminished Ki67 signals in the 
A

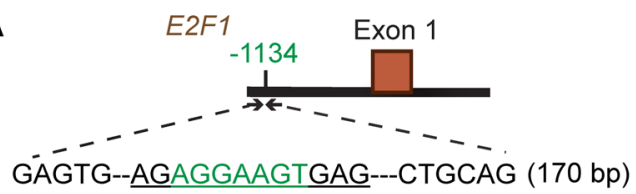

\section{C}

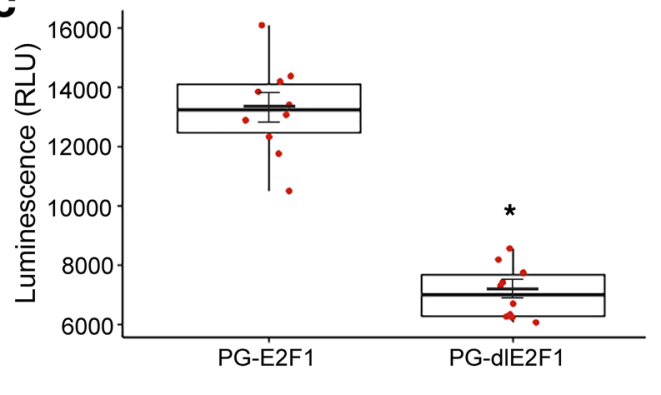

D
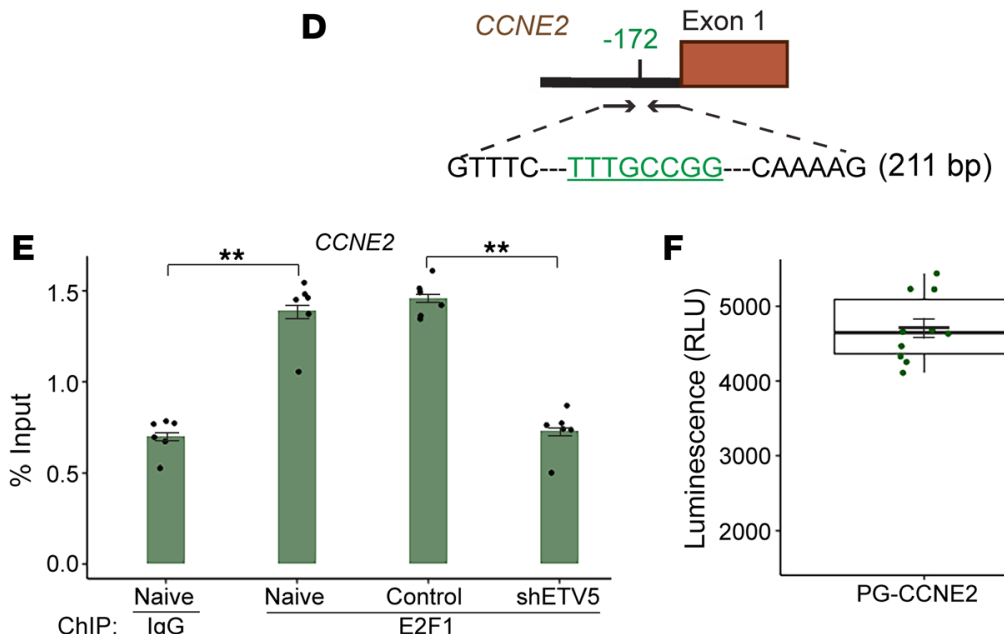

$\mathbf{F}$

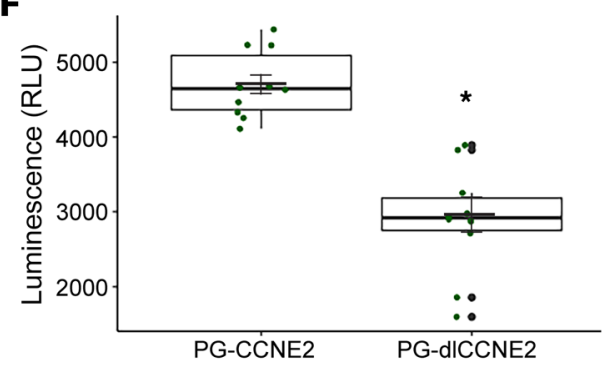

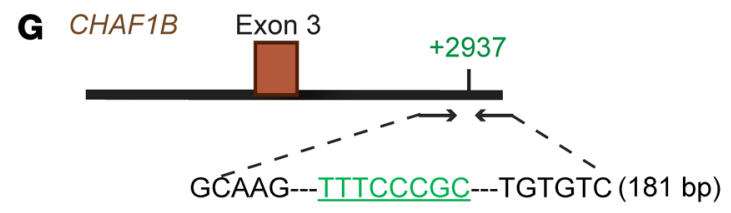

H

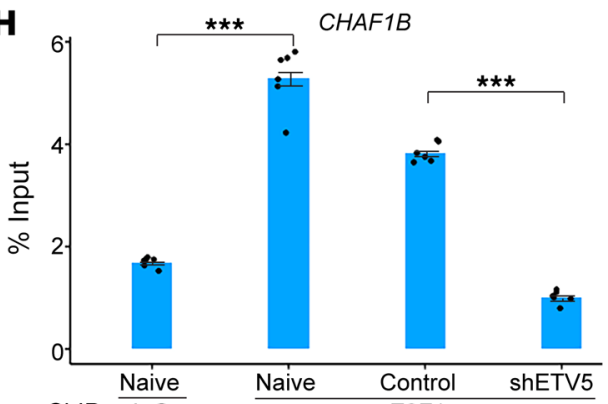
ChIP: $\frac{\text { IgG }}{\lg G}$
I

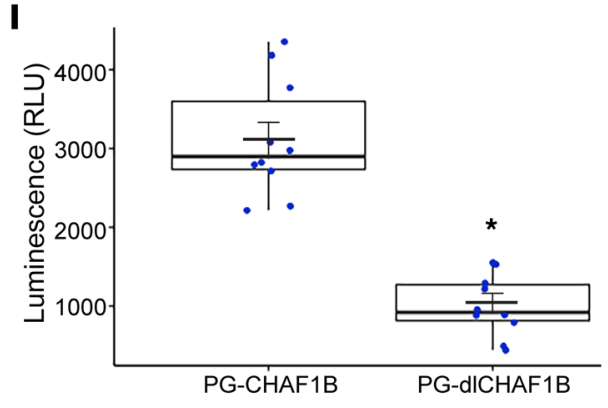

J

FGF/FGFR $\longrightarrow$ ETV4/ETV5 $\longrightarrow$ E2F1 $\longrightarrow$

Cell cycle

CHAF1A/B

Silenced chromatin
Figure 10. ETV5 and E2F1 genomic binding in SS cells. (A, D, and $\mathbf{G}$ )

Diagrams display the sequence and location of the putative binding motifs (green) of ETV5 on the E2F1 gene, and of E2F1 on the CCNE2 and CHAF1B genes. The diagrams also delineate the regions selected for $\mathrm{QPCR}$ amplification and subcloning in the PGL3 reporter vector. (B, E, and $\mathbf{H}$ ) Bar graphs show ETV5 binding to E2F1 (B) and E2F1 binding to CCNE2 and CHAF1B genes ( $\mathbf{E}$ and $\mathbf{H}$ ). Dots (12 per group in $\mathbf{B}$ and 6 per group in $\mathbf{E}$ and $\mathbf{H}$ ) represent values from 2 independent ChIP-qPCR experiments. IgG served as background control. IgG binding and ETV5 or E2F1 binding were quantified as percentage of input chromatin. Error bars indicate SEM. $P$ values $\left({ }^{*} P \leq 0.00009\right.$; ${ }^{* *} P<$ $\left.0.00001 ;{ }^{* *} P<0.00001\right)$ compare $\Delta \mathrm{Ct}$ averages of ETV 5 or E2F1 antibody versus IgG in naive cells and compare $\Delta$ Ct averages of ETV5 or E2F1 binding in shETV5-expressing cells versus control (TRC2) cells. The PCR primers used are described in Supplemental Methods. (C, F, and I) Box plots demonstrate endogenous transcriptional activity measured by luminescence (RLU) and driven by the binding regions of ETV5 (PG-E2F1, C) and E2F1 (PG-CCNE2, F; and PG-CHAF1B, I), subcloned in PGL3. The PG-dIE2F1, PG-dICCNE2, and PG-dICHAF1B lanes show transcriptional activity of the 3 regions lacking the binding motifs. The deleted sequences are underlined on the respective diagrams. The 10 dots in each lane represent individual values from 2 independent assays performed in 5 replicates. Crossbars indicate the mean, and error bars indicate SEM. $P$ values were measured by comparison of the average of each PG-dl group with that of the corresponding $P G$ vector. ${ }^{*} P<0.00001$. (J) Model for the ETV4/5 pathway in SS cells. 
BGJ398-treated tumors indicate that FGFR signaling generally functions as a proliferation pathway in SS.

Our results show that expression of ETV4 and ETV5 is regulated by FGFR signaling at the transcriptional level. Moreover, ETV4 and ETV5 synchronized activation in the mouse and human SS tumors clearly suggests that the two factors are coregulated. In development, ETV4 and ETV5 are often coexpressed, while ETV1 appears to be differently regulated (30). This might explain the apparent increase in ETV5 levels in the shFGFR1 and shFGFR3 cells (Figure 4, A and E), as it points to a likely compensation by ETV5-positive regulators upon ETV4 attenuation. This scenario is particularly pertinent in the shFGFR1/3 cells where FGFR2 signaling is still intact and capable of promoting ETV5 expression. Importantly, the extensive overlap in the individual transcription programs induced by ETV4 and ETV5 indicates analogous functions executed by both factors in SS. Nevertheless, the presence of ETV4 and ETV5 appears to be essential, since loss of either component leads to significant growth arrest and inability to initiate tumor formation. Thus, each factor constitutes a potential therapeutic target for SS in its own right, but given apparent feedback mechanisms, it is safe to state that a therapy regimen that completely incapacitates both factors will be needed to maximize inhibition of SS cell growth.

In CDS tumors, ETV4/5 upregulation occurs by direct binding of CIC-DUX4 to the gene promoters. Conversely, our data have demonstrated that $E T V 4 / 5$ derepression in SS is likely caused by lack of CIC binding due to FGFR/ERK signaling and undetectable ATXN1/ATXN1L, two events that are known to destabilize the CIC repressor complex. Further studies are needed to understand the details of this mechanism.

The coordinated downregulation of ERK1/2, DUSP6, and SPRY4 by BGJ398 is reminiscent of the oncogenic ERK-ETV5DUSP6/SPRY2 negative-feedback loop previously discovered as a driver of acute lymphocytic leukemia and a promoter of drug resistance (72). Our results indicate that an equivalent loop is located downstream of FGFR in SS cells. A recent report describes a similar mechanism where DUSP6 inhibition helps degrade the CICDUX4 oncoprotein by promoting CIC phosphorylation through MAPK-ERK activation (73). These findings suggest that targeting of DUSP6, though unintuitive, constitutes an appropriate addition or alternative for treating resistant SS tumors.

Downregulation of cell cycle and DNA repair mediators by ETV4 and ETV5 knockdown is consistent with the decrease in the proliferation marker Ki67 in the SS tumors treated with BGJ398. Importantly, it suggests that in SS, FGFR signaling controls proliferation via ETV4/5. ETV4 has previously been linked to cell cycle regulation by its promotion of cyclin D1 expression in pancreatic tumors (74). However, cyclin D1 was noticeably absent in our RNA-Seq screens, indicating that cell cycle regulation by the ETV factors is context specific. Moreover, the RNA-Seq, ETV5-ChIP, and endogenous reporter activity results indicate that cell cycle regulation by these factors in our systems likely occurs at the E2F level, thus explaining the downregulation of numerous E2F targets upon ETV4/5 depletion.

FSHD is an epigenetic disease, and a variety of chromatin silencers, such as CHAF1A/B, CBX1, EZH2, DNMT1, UHRF, and $\mathrm{RBBP} 7$, keep the $\mathrm{D} 4 \mathrm{Z} 4$ repeats and DUX4 in a heterochromatic state (65). Interestingly, most of the DUX4 repeat silencers are known E2F1 targets (61). Thus, their global downregulation in the shETV4/5 cells appears to be a result of E2F1 suppression. We focused our interest on the CHAF1A/B complex, as it was identified as a vital participant in DUX4 repression during embryonic development and in somatic cells. According to previous studies, CHAF1A/B inhibition alone could drive the global activation of DUX4 and its pathway $(34,65)$. Notably, we found that E2F1 binding to the $C H A F 1 B$ locus was significantly reduced in SS cells depleted of ETV5. The MBD3L (methyl-CpG binding domain protein 3-like) proteins are chromatin regulators that act as antagonists to NuRD-mediated silencing of the D4Z4 array (65). Thus, by directly activating the MBD3L family and the histone H3K9 demethylase KDM4E, DUX4 would ensure a permanent state of derepressed chromatin, and so create a positive-feedback loop for its own expression when ETV4 or ETV5 function is lost.

DUX4 expression in FSHD muscles activates a gene signature similar to that of the zygotic stage. Differentiated muscle cells seem to be intolerant to aberrant embryonic programs, hence their atrophy. However, the mechanism underlying this process is still unclear. Several studies addressing the question of DUX4 toxicity in somatic cells have presented a variety of mechanisms, such as activation of antiviral response, dsRNA and protein aggregation, p53, p21 (CDKN1A), and MYC-dependent induction of EGR1, among others. It is currently thought that more than one deathinducing pathway is involved in mediating DUX4 effects $(35,59$, $60,75)$. In our systems, ETV4/5 knockdown and DUX4 both lead to CDKN1A and EGR1 stimulation and cell death, and through this process, derepressed DUX4 likely compounds the cell cycle arrest caused by E2F1 suppression. Notably, a previous study has reported on EGR1 repression by the SS18-SSX fusion in SS cells (76). Further investigations will clarify whether a link exists between the several regulators that appear to converge on EGR1 in SS.

The SYO-1 and HS-SY-II cells carry the SS18-SSX2 and SS18SSX1 translocations, respectively. The 2 fusions represent the most frequent rearrangements in SS. Therefore, the biological findings in the 2 cell lines presented in these studies are of general relevance to SS. Uncovering the tumorigenic axis that links FGFR signaling to the ETV4/5 factors and the DUX4 program in SS carries wide significance. In addition to demonstrating the crucial role FGFR signaling plays in SS development, we have identified ETV4 and ETV5 as drivers of the cancer, and potential targets for future SS therapies. Finally, the surprising finding that DUX4 appears to act as a tumor suppressor in this sarcoma is contrary to the recent discovery that DUX4 functions as an oncogene in several malignancies (43), more particularly by converting CIC to an activator of the ETV4/5 oncogenes in CDS. With these opposite effects, we could conjecture that the mesenchymal progenitor background of SS tumors is a likely factor in influencing DUX4 behavior. Further mechanistic studies are needed to clarify this important point.

Altogether, our studies have uncovered a pathogenic link between FGFR signaling, ETV4/5, and the embryonic DUX4 pathway in SS. In addition to FGFR inhibition and ETV4/5 block, these findings highlight DUX4 activation by specific epigenetic modulators as an additional tier for targeting SS and other human cancers in which these pathways are active, and which would thus benefit from DUX4-induced atrophy. 


\section{Methods}

Cells. The SYO-1, HS-SY-II, and C2C12 cell lines were grown in DMEM supplemented with $10 \%$ FBS and $1 \times$ penicillin/streptomycin (PS). The SYO-1 and HS-SY-II cells were provided by Marc Ladanyi (Memorial Sloan Kettering Cancer Center, New York, New York, USA), and the $\mathrm{C} 2 \mathrm{C} 12$ cells were obtained from the American Type Culture Collection. The SS cells were authenticated through detection of the SS18-SSX fusion and grown on collagen-coated plates. The CDS-X1 cells (50) were maintained in RPMI 1640 plus $10 \%$ FBS and $1 \times$ PS. The CDS-S2 cells (51) were maintained in DMEM/F-12-GlutaMAX (Gibco) plus 10\% FBS. The CDS cells were authenticated by detection of the CIC-DUX4 fusion oncoprotein (Supplemental Figure 4B).

Genetic and xenograft mouse models. Mice were acquired, housed, and bred following an approved protocol (19-184LF) and rules set by the Institutional Animal Care and Use Committee at the University of Miami. The triple-knockout Fgfr $1,2,3^{f / f l}$ mice were obtained from the Ornitz laboratory (Washington University) $(46,77,78)$. The SSM2 ${ }^{+}$and $M y f 5-c r e^{+}$transgenic mice were obtained from the Capecchi laboratory (University of Utah). C57BL6/J mice used for the generation of $F g f r 1^{f l / f l}, F g f r 2^{f / / l l}$, and $F g f 3^{f / f l}$ (scheme not shown) and the NU/J mice were acquired from The Jackson Laboratory.

Genotyping of SSM2 ${ }^{+}$and Myf5-cre $e^{+}$mice was performed as reported previously (7). Genotyping of Fgfr-floxed mice was conducted by PCR as recommended by the Ornitz laboratory, with the GoTaq Flexi polymerase kit (Promega). The PCR conditions were as follows: $94^{\circ} \mathrm{C}$ for 3 minutes; 35 cycles of $94^{\circ} \mathrm{C}$ for 30 seconds and $68^{\circ} \mathrm{C}$ for 3 minutes; $72^{\circ} \mathrm{C}$ for 5 minutes. The Fgfr primers (Ornitz laboratory) used for genotyping were: Fgfr1 forward, $5^{\prime}$-CCAGTAACTGTACCAATGAGCTGTAAGCAT-3'; Fgfr1 reverse, 5'-TGCCCACCATGCTCCTGCTTCCTTCAGAGC-3'; Fgfr2 forward, 5'-TTCCTGTTCGACTATAGGAGCAACAGGCGG-3'; Fgfr2 reverse, 5'-GAGAGCAGGGTGCAAGAGGCGACCAGTCAG-3'; Fgfr3 forward, 5'-GCTCCCTGTCCTGCCTCGTGTCTCCTAG-3'; Fgfr3 reverse, 5'-AGGACAAATTGGTACCATACAACGTG-3'. Detection, counting, and measurement of tumors in the SM2, SMF1, SMF2, and SMF3 and in the BGJ398-treated SM2 mice were performed after euthanasia as previously described (7).

The xenograft SS models (BGJ398-treated tumors and shETV4/5 tumors) were generated with subcutaneous injection of $8 \times 10^{6}$ SYO- 1 cells in $100 \mu \mathrm{L}$ of $1 \times$ DPBS in Matrigel, in the right flank of NU/J mice. Visible tumors in all models were measured with a digital caliper.

Further information and experimental details are provided in Supplemental Methods.

Statistics. All graphs presented in the paper (dot plots, box plots, bar graphs) were drawn in RStudio, with the exception of the BGJ398 dose-response curves in Figure 2, A and B, which were generated in Microsoft Excel.

In graphs where 3 or more experimental groups were included namely those of Figure 4, B, D, and F; Figure 5, H-J; Figure 6, C, D, F, and G; Figure 9, C-E; and Supplemental Figure 2-1-way ANOVA was used for comparison of multiple means. $P$ values calculated from ANOVA $F$ ratios showing significant difference were followed by 2 -tailed $t$ tests to compare means between 2 groups, as specified in the figure legends. The mean (average of values), SEM, and SD were calculated in R or Excel. $P$ values less than or equal to 0.05 were considered significant in all statistical tests used.
In the RT-qPCR graphs, individual Ct (threshold cycle, also known as $\mathrm{Cq}$ or $\triangle \mathrm{R}$ ) values were normalized against $G A P D H$ average $\mathrm{Ct}$, and SDs between 2 experimental groups were derived from average $\Delta \Delta \mathrm{Ct}$. Fold change was calculated as FC $=2^{-\Delta \Delta C t}$. In the ChIP-qPCR graphs, the average $\mathrm{Ct}$ of input chromatin was adjusted to $2 \%$ of total chromatin. $\Delta \mathrm{Cts}$ of the antibody were adjusted to the new average $\mathrm{Ct}$ of the input. Percentage input of individual antibody $\Delta$ Cts was calculated as $100 \times 2^{\Delta \mathrm{Ct}} . P$ values were derived from SDs based on average $\Delta \mathrm{Cts}$ of experimental groups.

Intensities of PCR products (RT-PCR) and protein bands (immunoblots) were measured using Image software (NIH) as follows: Background intensity was subtracted from absolute numbers, and then the average intensity of the respective loading controls - actin lanes in immunoblots and GAPDH lanes in RT-PCR gels - was calculated. Ratios of individual DNA or protein band intensities over average intensity of loading controls were measured, and fold change in gene transcription or protein expression was determined by comparison of the ratios of band intensities with those of the experimental controls (adjusted to 1).

Study approval. The mouse studies were approved by the Institutional Animal Care and Use Committee at the University of Miami under protocol 19-184LF. Acquisition and analysis of human SS tumors were approved by University of Miami Hospital and Clinics under institutional research board protocol IRB 20121060.

\section{Author contributions}

JD, YB, LL, XS, ZJ, MB, and JEE performed experiments and data analysis. DAK, MK, MRC, JMP, LC, TK, and DMO provided key reagents. JD, JCT, DMO, and JEE conceived the experimental plan and wrote the manuscript.

\section{Acknowledgments}

We thank Alex Paul Sanchez-Covarrubias and the Sophia George Laboratory (University of Miami) for use of the real-time PCR machine and technical support, and Agata Levay (University of Miami) for the PGL3 vector. We thank Beatrice Victoria Maria and Theo Sing for critical guidance. This work was supported by the National Cancer Institute of the NIH under award P30CA240139 (Sylvester Cancer Center/University of Miami), NIH grant HL111190 (to DMO), and NIH grant 1S10OD023579-01 for the VS120 Slide Scanner housed at the University of Miami Miller School of Medicine Analytical Imaging Core Facility.

Address correspondence to: Josiane E. Eid, Department of Medicine, University of Miami Miller School of Medicine, 1120 NW 14th Street, Miami, Florida 33136, USA. Phone: 305.243.1197; Email:jee64@med.miami.edu.

\section{ZJ's present address is: M2Gen, Tampa, Florida, USA.}

DAK's present address is: Pathology and Laboratory Medicine, Dartmouth-Hitchcock Medical Center, Lebanon, New Hampshire, USA; and Geisel School of Medicine at Dartmouth, Hanover, New Hampshire, USA.

MK's present address is: Department of Hematopathology, MD Anderson Cancer Center, Houston, Texas, USA. 
1. El Beaino M, et al. Synovial sarcoma: a complex disease with multifaceted signaling and epigenetic landscapes. Curr Oncol Rep. 2020;22(12):124.

2. Stacchiotti S, Van Tine BA. Synovial sarcoma: current concepts and future perspectives. J Clin Oncol. 2018;36(2):180-187.

3. Kadoch C, Crabtree GR. Reversible disruption of mSWI/SNF (BAF) complexes by the SS18SSX oncogenic fusion in synovial sarcoma. Cell. 2013;153(1):71-85.

4. Banito A, et al. The SS18-SSX oncoprotein hijacks KDM2B-PRC1.1 to drive synovial sarcoma. Cancer Cell. 2018;33(3):527-541.

5. Hale R, et al. Epigenetic targets in synovial sarcoma: a mini-review. Front Oncol. 2019;9:1078.

6. Garcia $\mathrm{CB}$, et al. Reprogramming of mesenchymal stem cells by the synovial sarcomaassociated oncogene SYT-SSX2. Oncogene. 2012;31(18):2323-2334.

7. Barham W, et al. Targeting the Wnt pathway in synovial sarcoma models. Cancer Discov. 2013;3(11):1286-1301.

8. Ishibe $\mathrm{T}$, et al. Disruption of fibroblast growth factor signal pathway inhibits the growth of synovial sarcomas: potential application of signal inhibitors to molecular target therapy. Clin Cancer Res. 2005;11(7):2702-2712.

9. Ornitz DM, Itoh $\mathrm{N}$. The fibroblast growth factor signaling pathway. Wiley Interdiscip Rev Dev Biol. 2015;4(3):215-266.

10. Ornitz DM, Marie PJ. Fibroblast growth factor signaling in skeletal development and disease. Genes Dev. 2015;29(14):1463-1486.

11. Turner N, Grose R. Fibroblast growth factor signalling: from development to cancer. Nat Rev Cancer. 2010;10(2):116-129.

12. Wöhrle S, et al. Fibroblast growth factor receptors as novel therapeutic targets in SNF5deleted malignant rhabdoid tumors. PLoS One. 2013;8(10):e77652.

13. Yin $Y$, et al. Rapid induction of lung adenocarcinoma by fibroblast growth factor 9 signaling through FGF receptor 3. Cancer Res. 2013;73(18):5730-5741.

14. Yin Y, et al. Fibroblast growth factor 9 regulation by microRNAs controls lung development and links DICER1 loss to the pathogenesis of pleuropulmonary blastoma. PLoS Genet. 2015;11(5):e1005242.

15. Agelopoulos K, et al. Deep sequencing in conjunction with expression and functional analyses reveals activation of FGFR1 in Ewing sarcoma. Clin Cancer Res. 2015;21(21):4935-4946.

16. Hegab AE, et al. Effect of FGF/FGFR pathway blocking on lung adenocarcinoma and its cancer-associated fibroblasts. J Pathol. 2019;249(2):193-205.

17. Li F, et al. FGFR-mediated reactivation of MAPK signaling attenuates antitumor effects of imatinib in gastrointestinal stromal tumors. Cancer Discov. 2015;5(4):438-451.

18. Sizemore GM, et al. The ETS family of oncogenic transcription factors in solid tumours. Nat Rev Cancer. 2017;17(6):337-351.

19. Diez Del Corral R, Morales AV. The multiple roles of FGF signaling in the developing spinal cord. Front Cell Dev Biol. 2017;5:58.

20. Song HW, Wilkinson MF. Transcriptional control of spermatogonial maintenance and differentiation. Semin Cell Dev Biol. 2014;30:14-26.

21. Herriges JC, et al. FGF-regulated ETV transcription factors control FGF-SHH feedback loop in lung branching. Dev Cell. 2015;35(3):322-332.

22. Zhang Z, et al. FGF-regulated Etv genes are essential for repressing Shh expression in mouse limb buds. Dev Cell. 2009;16(4):607-613.

23. Specht $\mathrm{K}$, et al. Distinct transcriptional signature and immunoprofile of CIC-DUX4 fusion-positive round cell tumors compared to EWSR1rearranged Ewing sarcomas: further evidence toward distinct pathologic entities. Genes Chromosomes Cancer. 2014;53(7):622-633.

24. Padul V, et al. ETV/Pea3 family transcription factor-encoding genes are overexpressed in CIC-mutant oligodendrogliomas. Genes Chromosomes Cancer. 2015;54(12):725-733.

25. Le Guellec S, et al. ETV4 is a useful marker for the diagnosis of CIC-rearranged undifferentiated round-cell sarcomas: a study of 127 cases including mimicking lesions. Mod Pathol. 2016;29(12):1523-1531.

26. Okimoto RA, et al. Inactivation of Capicua drives cancer metastasis. Nat Genet. 2017;49(1):87-96.

27. Okimoto RA, et al. CIC-DUX4 oncoprotein drives sarcoma metastasis and tumorigenesis via distinct regulatory programs. JClin Invest. 2019;129(8):3401-3406.

28. Monge M, et al. ERM/ETV5 up-regulation plays a role during myometrial infiltration through matrix metalloproteinase-2 activation in endometrial cancer. Cancer Res. 2007;67(14):6753-6759.

29. Firlej V, et al. Reduced tumorigenesis in mouse mammary cancer cells following inhibition of Pea3- or Erm-dependent transcription. J Cell Sci. 2008;121(Pt 20):3393-3402.

30. Oh S, et al. ETV1, 4 and 5: an oncogenic subfamily of ETS transcription factors. Biochim Biophys Acta. 2012;1826(1):1-12.

31. Aytes A, et al. ETV4 promotes metastasis in response to activation of PI3-kinase and Ras signaling in a mouse model of advanced prostate cancer. Proc Natl Acad Sci US A. 2013;110(37):E3506-E3515.

32. Wang B, et al. ATXN1L, CIC, and ETS transcription factors modulate sensitivity to MAPK pathway inhibition. Cell Rep. 2017;18(6):1543-1557.

33. De Iaco A, et al. DUX-family transcription factors regulate zygotic genome activation in placental mammals. Nat Genet. 2017;49(6):941-945.

34. Hendrickson PG, et al. Conserved roles of mouse DUX and human DUX4 in activating cleavage-stage genes and MERVL/HERVL retrotransposons. Nat Genet. 2017;49(6):925-934.

35. Campbell AE, et al. Facioscapulohumeral dystrophy: activating an early embryonic transcriptional program in human skeletal muscle. Hum Mol Genet. 2018;27(r2):R153-R162.

36. Himeda CL, Jones PL. The genetics and epigenetics of facioscapulohumeral muscular dystrophy. Annu Rev Genomics Hum Genet. 2019;20:265-291.

37. Das S, Chadwick BP. Influence of repressive histone and DNA methylation upon D4Z4 transcription in non-myogenic cells. PLoS One. 2016;11(7):e0160022.

38. Geng LN, et al. DUX4 activates germline genes, retroelements, and immune mediators: implications for facioscapulohumeral dystrophy. Dev Cell. 2012;22(1):38-51.

39. Yao Z, et al. DUX4-induced gene expression is the major molecular signature in FSHD skeletal muscle. Hum Mol Genet. 2014;23(20):5342-5352.

40. Kawamura-Saito M, et al. Fusion between CIC and DUX4 up-regulates PEA3 family genes in Ewing-like sarcomas with $\mathrm{t}(4 ; 19)(\mathrm{q} 35 ; \mathrm{q} 13)$ translocation. Hum Mol Genet. 2006;15(13):2125-2137.

41. Italiano A, et al. High prevalence of CIC fusion with double-homeobox (DUX4) transcription factors in EWSR1-negative undifferentiated small blue round cell sarcomas. Genes Chromosomes Cancer. 2012;51(3):207-218.

42. Yasuda T, et al. Recurrent DUX4 fusions in B cell acute lymphoblastic leukemia of adolescents and young adults. Nat Genet. 2016;48(5):569-574.

43. Chew GL, et al. DUX4 suppresses MHC class I to promote cancer immune evasion and resistance to checkpoint blockade. Dev Cell. 2019;50(5):658-671.

44. Haldar M, et al. A conditional mouse model of synovial sarcoma: insights into a myogenic origin. Cancer Cell. 2007;11(4):375-388.

45. Dong Z, et al. FGF2-induced STAT3 activation regulates pathologic neovascularization. Exp Eye Res. 2019;187:107775.

46. Meyer M, et al. Mouse genetics identifies unique and overlapping functions of fibroblast growth factor receptors in keratinocytes. J Cell Mol Med. 2020;24(2):1774-1785.

47. Guagnano V, et al. Discovery of 3-(2,6-dichloro3,5-dimethoxy-phenyl)-1-\{6-[4-(4-ethyl-piperazin1-yl)-phenylamino]-pyrimidin-4-yl\}-1-methylurea (NVP-BGJ398), a potent and selective inhibitor of the fibroblast growth factor receptor family of receptor tyrosine kinase. JMed Chem. 2011;54(20):7066-7083.

48. Guagnano V, et al. FGFR genetic alterations predict for sensitivity to NVP-BGJ398, a selective pan-FGFR inhibitor. Cancer Discov. 2012;2(12):1118-1133.

49. Porta R, et al. FGFR a promising druggable target in cancer: Molecular biology and new drugs. Crit Rev Oncol Hematol. 2017;113:256-267.

50. Oyama R, et al. Generation of novel patientderived CIC- DUX4 sarcoma xenografts and cell lines. Sci Rep. 2017;7(1):4712.

51. Yoshimatsu Y, et al. Establishment and characterization of NCC-CDS2-C1: a novel patientderived cell line of CIC-DUX4 sarcoma. Hum Cell. 2020;33(2):427-436.

52. Wong D, Yip S. Making heads or tails - the emergence of capicua (CIC) as an important multifunctional tumour suppressor. J Pathol. 2020;250(5):532-540.

53. Forés $\mathrm{M}$, et al. A new mode of DNA binding distinguishes Capicua from other HMG box factors and explains its mutation patterns in cancer. PLoS Genet. 2017;13(3):e1006622.

54. Antonescu CR, et al. Sarcomas with CIC-rearrangements are a distinct pathologic entity with aggressive outcome: a clinicopathologic and molecular study of 115 cases. Am J Surg Pathol. 2017;41(7):941-949.

55. Nielsen TO, et al. Molecular characterisation of soft tissue tumours: a gene expression study. 
Lancet. 2002;359(9314):1301-1307.

56. Francis $P$, et al. Diagnostic and prognostic gene expression signatures in 177 soft tissue sarcomas: hypoxia-induced transcription profile signifies metastatic potential. BMC Genomics. 2017;8:73.

57. El Beaino M, et al. Diagnostic value of TLE1 in synovial sarcoma: a systematic review and meta-analysis. Sarcoma. 2020;2020:7192347.

58. Jagannathan S, et al. Model systems of DUX4 expression recapitulate the transcriptional profile of FSHD cells. Hum Mol Genet. 2016;25(20):4419-4431.

59. Xu H, et al. Dux4 induces cell cycle arrest at G1 phase through upregulation of p21 expression. Biochem Biophys Res Commun. 2014;446(1):235-240.

60. Shadle SC, et al. DUX4-induced dsRNA and MYC mRNA stabilization activate apoptotic pathways in human cell models of facioscapulohumeral dystrophy. PLoS Genet. 2017;13(3):e1006658.

61. Fischer M, et al. Integration of TP53, DREAM, MMB FOXM1, and RB-E2F target gene analyses identifies cell cycle gene regulatory networks. Nucleic Acids Res. 2016;44(13):6070-6086.

62. Johnson M, et al. Identification of head and neck cancer subtypes based on human papillomavirus presence and E2F-regulated gene expression. mSphere. 2018;3(1):e00580-17.

63. Hollenhorst PC, et al. Oncogenic ETS proteins mimic activated RAS/MAPK signaling in prostate cells. Genes Dev. 2011;25(20):2147-2157.

64. Safran M, et al. GeneCards Version 3: the human gene integrator. Database (Oxford). 2010;2010:baq020.

65. Campbell AE, et al. NuRD and CAF-1-mediated silencing of the D4Z4 array is modulated by DUX4-induced MBD3L proteins. Elife. 2018;7:e31023.

66. Neuman E, et al. Transcription of the E2F-1 gene is rendered cell cycle dependent by E2F DNA-binding sites within its promoter. Mol Cell Biol. 1994;14(10):6607-6615.

67. Naka N, et al. Synovial sarcoma is a stem cell malignancy. Stem Cells. 2010;28(7):1119-1131.

68. Eid JE, Garcia CB. Reprogramming of mesenchymal stem cells by oncogenes. Semin Cancer Biol. 2015;32:18-31.

69. Garcia CB, et al. Genome-wide recruitment to Polycomb-modified chromatin and activity regulation of the synovial sarcoma oncogene SYTSSX2. BMC Genomics. 2012;13:189.

70. Ford-Perriss M, et al. Fibroblast growth factors in the developing central nervous system. Clin Exp Pharmacol Physiol. 2001;28(7):493-503.

71. Yin Y, Ornitz DM. FGF9 and FGF10 activate distinct signaling pathways to direct lung epithelial specification and branching. Sci Signal. 2020;13(621): eaay4353.

72. Shojaee S, et al. Erk negative feedback control enables pre-B cell transformation and represents a therapeutic target in acute lymphoblastic leukemia. Cancer Cell. 2015;28(1):114-128.

73. Lin YK, et al. Negative MAPK-ERK regulation sustains CIC-DUX4 oncoprotein expression in undifferentiated sarcoma. Proc Natl Acad Sci U S A. 2020;117(34):20776-20784.

74. Tyagi N, et al. ETV4 facilitates cell-cycle progression in pancreatic cells through transcriptional regulation of cyclin D1. Mol Cancer Res. 2018;16(2):187-196.

75. Rickard AM, et al. Endogenous DUX4 expression in FSHD myotubes is sufficient to cause cell death and disrupts RNA splicing and cell migration pathways. Hum Mol Genet. 2015;24(20):5901-5914.

76. Su L, et al. Deconstruction of the SS18-SSX fusion oncoprotein complex: insights into disease etiology and therapeutics. Cancer Cell. 2012;21(3):333-347.

77. Trokovic N, et al. Fgfr1 regulates patterning of the pharyngeal region. Genes Dev. 2003;17(1):141-153.

78. Su N, et al. Generation of Fgfr3 conditional knockout mice. Int J Biol Sci. 2010;6(4):327-332.

79. Vichai V, Kirtikara K. Sulforhodamine B colorimetric assay for cytotoxicity screening. Nat Protoc. 2006;1(3):1112-1116.

80. FastQC. Version 0.11.9. Andrews S; Babraham Bioinformatics; 2019. http://www.bioinformatics. babraham.ac.uk/projects/fastqc/. Accessed May 12, 2021.

81. Dobin A, et al. STAR: ultrafast universal RNA-seq aligner. Bioinformatics. 2013;29(1):15-21.

82. Li B, Dewey CN. RSEM: accurate transcript quantification from RNA-Seq data with or without a reference genome. BMC Bioinformatics. 2011;12:323.

83. Pretto D, et al. The synovial sarcoma translocation protein SYT-SSX2 recruits beta-catenin to the nucleus and associates with it in an active complex. Oncogene. 2006;25(26):3661-3669.

84. Boyer LA, et al. Core transcriptional regulatory circuitry in human embryonic stem cells. Cell. 2005;122(6):947-956.

85. Gaillard MC, et al. Analysis of the 4q35 chromatin organization reveals distinct long-range interactions in patients affected with Facio-ScapuloHumeral Dystrophy. Sci Rep. 2019;9(1):10327.

86. Ferreboeuf M, et al. DUX4 and DUX4 downstream target genes are expressed in fetal FSHD muscles. Hum Mol Genet. 2014;23(1):171-181. 